\title{
SHC-1/p52Shc targets the insulin/IGF-1 and JNK signaling pathways to modulate life span and stress response in C. elegans
}

\author{
Elke Neumann-Haefelin, ${ }^{1,2,3}$ Wenjing Qi, ${ }^{1,2}$ Elisabeth Finkbeiner, ${ }^{1,2}$ Gerd Walz, ${ }^{3}$ \\ Ralf Baumeister, ${ }^{1,2,4,5}$ and Maren Hertweck ${ }^{1}$ \\ ${ }^{1}$ Bioinformatics and Molecular Genetics (Faculty of Biology), Center for Biochemistry and Molecular Cell Research \\ (Faculty of Medicine), University of Freiburg, D-79104 Freiburg, Germany; ${ }^{2}$ Center for Systems Biology (ZBSA), \\ University of Freiburg, D-79104 Freiburg, Germany; ${ }^{3}$ Renal Division, University Hospital Freiburg, D-79106 Freiburg, \\ Germany; ${ }^{4}$ FRIAS School of Life Sciences (LIFENET), D-79104 Freiburg, Germany
}

\begin{abstract}
Correlative evidence links stress, accumulation of oxidative cellular damage, and aging in several species. Genetic studies in species ranging from yeast to mammals revealed several pathways regulating stress response and life span, including caloric intake, mitochondrial respiration, insulin/IGF-1 (IIS), and JNK (c-Jun N-terminal kinase) signaling. How IIS and JNK signaling cross-talk to defend against diverse stressors contributing to aging is of critical importance but, so far, only poorly understood. In this study, we demonstrate that the adaptor protein SHC-1, the Caenorhabditis elegans homolog of human p52She, coordinates mechanisms of stress response and aging. Using genetic and biochemical approaches, we discover that SHC-1 not only opposes IIS but also activates JNK signaling. Loss of shc-1 function results in accelerated aging and enhanced sensitivity to heat, oxidative stress, and heavy metals, whereas expression of human p52She rescues the shc-1 mutant phenotype. SHC-1 acts upstream of the insulin/IGF receptor DAF-2 and the PI3 kinase AGE-1 and directly interacts with DAF-2. Moreover, SHC-1 activates JNK signaling by binding to MEK-1 kinase. Both aspects converge on controlling the nuclear translocation and activation of the FOXO transcription factor DAF-16. Our findings establish C. elegans SHC-1 as a critical scaffold that directly cross-connects the two parallel JNK and IIS pathways and help to explain how these signaling cascades cooperate to ascertain normal stress response and life span in C. elegans.
\end{abstract}

[Keywords: SHC-1/p52Shc; C. elegans; aging; stress response; insulin; JNK]

Supplemental material is available at http://www.genesdev.org.

Received March 6, 2008; revised version accepted July 28, 2008.

There is considerable evidence that aging is regulated by distinct evolutionarily conserved mechanisms and pathways, including insulin/IGF-1 signaling (IIS) (Kenyon et al. 1993; Gottlieb and Ruvkun 1994; Clancy et al. 2001; Tatar et al. 2001; Bluher et al. 2003), JNK (c-Jun N-terminal kinase) signaling (Wang et al. 2003; Oh et al. 2005), caloric intake (Lin et al. 2000; Houthoofd et al. 2002), mitochondrial respiration (Lakowski and Hekimi 1998; Lee et al. 2003), and signaling from the germline (Hsin and Kenyon 1999; Arantes-Oliveira et al. 2002).

Shc-like proteins have been identified from nematodes to humans, which suggests that their role might also be conserved in evolution (Luzi et al. 2000). The mamma-

${ }^{5}$ Corresponding author.

E-MAIL baumeister@celegans.de; FAX 49-761-2038352.

Article is online at http://www.genesdev.org/cgi/doi/10.1101/gad.478408. lian Shc gene encodes three proteins of 46, 52, and 66 $\mathrm{kDa}$. All Shc proteins share domain arrangements consisting of an N-terminal phosphotyrosine-binding (PTB) domain and a C-terminal Src-homology 2 (SH2) domain. Both domains have the ability to bind tyrosine-phosphorylated proteins, but they differ in their phosphopeptide-binding specificities (Zhou et al. 1995). Only p66Shc is characterized by an additional $\mathrm{N}$-terminal $\mathrm{CH}$-like domain (Migliaccio et al. 1999; Gertz et al. 2008).

Shc proteins were initially identified as molecular adaptors that function as signal transducers in receptor tyrosine kinase signaling. Distinct She variants probably have different functions and are also localized in distinct subcellular compartments. p66 is predominantly mitochondrial, p52 is cytoplasmic, and p46 was observed in nuclear fractions (Yukimasa et al. 2005). p52/46Shc contributes to Ras-dependent mitogen-activated protein ki- 
nase (MAPK) activation (Pelicci et al. 1992). However, MAPK activation and the involvement of p52/46Shc in mitogenic processes are not the sole functions of Shc. Upon phosphorylation, p52/46Shc can serve as a scaffold for downstream signaling effectors in response to a variety of growth factors (including EGF, PDGF, and IGF), but it is currently unknown whether p52/46Shc contributes to the regulation of stress response and life span. So far, this has been considered to be a unique property of p66Shc.

The mouse $p 66 s h c$ gene has been the first gerontogene described in a mammalian organism. p66Shc ${ }^{-/-}$mice experience a $30 \%$ extension in life span and increased resistance to oxidative stress (Migliaccio et al. 1999). As a consequence, Shc protein function has been proposed to link oxidative stress, apoptosis, and aging in mammals (Giorgio et al. 2005; Pinton et al. 2007).

From Caenorhabditis elegans to higher vertebrates, signaling through the insulin/IGF-1 receptor DAF-2 is one of the most important pathways to regulate stress response and to affect life span. Upon ligand binding, phosphorylation of the insulin receptor activates the PI3kinase AGE-1 (Morris et al. 1996). The major effector of AGE-1 is PDK-1, which, in turn, activates a multimeric complex formed by AKT-1/AKT-2/SGK-1 (Paradis and Ruvkun 1998; Paradis et al. 1999; Hertweck et al. 2004). In both, worms and vertebrates, IIS antagonizes FOXO transcription factors. In C. elegans, inactivation of the FOXO transcription factor DAF-16 involves AKT/SGKmediated phosphorylation and subsequent cytoplasmic retention of DAF-16 (Lin et al. 1997; Lee et al. 2001). Reduction of daf-2 gene activity or that of other genes in the IIS pathway that are positively regulated by daf-2 substantially prolongs life span and enhances oxidative stress resistance. daf-16 is essential for this phenotype of IIS mutants, indicating that DAF-16 is the major downstream effector of the IIS pathway (Ogg et al. 1997).

Additional input into FOXO/DAF-16 regulation is conferred by JNK-1, a member of the MAPK superfamily. The JNK pathway is highly conserved in evolution and has been implicated in a variety of biological functions, including development, apoptosis, and response to environmental stress (for review, see Davis 2000). Generally, extracellular signals activate MAPKs through dual-specific MAPK kinases (MAPKK). In mammalian cells, evidence suggests that the MAPKK MKK7 specifically activates JNK. In C. elegans, the JNK pathway consists of the Jnk homolog JNK-1 and two MKK7-type MAPKKs, JKK-1 and MEK-1 (Kawasaki et al. 1999; Koga et al. 2000; Villanueva et al. 2001). Genetic studies demonstrate that $j k k-1$ and $j n k-1$ regulate life span and stress response. JKK-1 specifically stimulates the kinase activity of JNK-1 (Kawasaki et al. 1999). Activation of JNK-1 results in increased life span and tolerance for oxidative and thermal stress (Oh et al. 2005). JNK-1 extends life span by promoting phosphorylation-dependent nuclear translocation of DAF-16, indicating that the IIS and JNK pathways act in parallel to converge on DAF-16. Disruption of the MAPKK gene mek-1 results in hypersensitivity to heavy metals and enhanced susceptibility to pathogenic bacteria and starvation, suggesting that MEK-1 also plays a central, yet distinct role in stress response (Koga et al. 2000; Kim et al. 2004).

We report here that the adaptor protein SHC-1 mediates a novel cross-talk between IIS and JNK signaling pathways in C. elegans, and we show that shc-1 is the functional homolog of human p52Shc. SHC-1 coordinates mechanisms of stress response and aging, functions that previously have been exclusively attributed to p66Shc in mammals. Loss of shc-1 function results in accelerated aging and enhanced sensitivity to heat, oxidative, and heavy metal stress. Our data reveal that SHC-1 not only represses IIS through direct interaction with the DAF-2/insulin receptor, but also activates JNK signaling by binding to MEK-1. Our findings establish SHC-1/p52Shc as a linker of the two parallel IIS and JNK pathways and help to explain how these signaling cascades cooperate to modulate the activity of the FOXO transcription factor DAF-16. The two observations that the major components of both mechanisms are encoded in the genomes of most multicellular animals and that defective $C$. elegans shc-1 can be rescued by transgenic expression of human p52Shc suggest an evolutionary conservation of $s h c-1 / \mathrm{p} 52 \mathrm{Shc}$ function.

\section{Results}

\section{Identification of SHC proteins in C. elegans}

In a search for Shc-like proteins in C. elegans, we identified one protein with high sequence similarity to human p52Shc (Luzi et al. 2000) and named the corresponding gene shc-1 (corresponding to F54A5.3). C. elegans SHC-1 displays the N- to C-terminal PTB-SH2 domain structure conserved in all Shc proteins but does not contain the additional $\mathrm{N}$-terminal $\mathrm{CH} 2$ region present in p66Shc (Fig. 1A,B) that functions as a redox module for apoptosis initiation (Gertz et al. 2008).

Using translational GFP fusions that rescue the shc-1 mutant phenotype (see below), we determined the expression pattern of shc-1. In agreement with p52Shc in mammalian cells (Pelicci et al. 1992), we observed broad expression of $s h c-1:: g f p$ in many tissues. GFP staining was observed in head and tail neurons, hypodermis, pharynx, vulva muscles, and intestine throughout all stages of post-embryonic development and adulthood (Fig. 1D-H). In most cells, SHC-1 ::GFP localized to both the cytoplasm and the nucleus. A portion of the protein seems to be associated with the cytoplasmic membrane. Interestingly, in intestinal cells SHC-1 ::GFP strongly localized to the nucleus (Fig. $1 \mathrm{H}$ ), and in worms this is the prominent tissue where the activity of the IIS effector DAF-16 is required (for review, see Piper et al. 2008).

To analyze the function of shc-1, we characterized a deletion allele of the $s h c-1$ gene, kindly provided by the C. elegans Gene Knockout Consortium. We determined by DNA sequencing that shc-1(ok198) contains a deletion of 1177 base pairs (bp) (including exon 4). RT-PCR demonstrated that this deletion results in an erroneous splicing from exon 3 to 5 with a frameshift in exon 5 and an early translational stop at position 282 (data not 
A

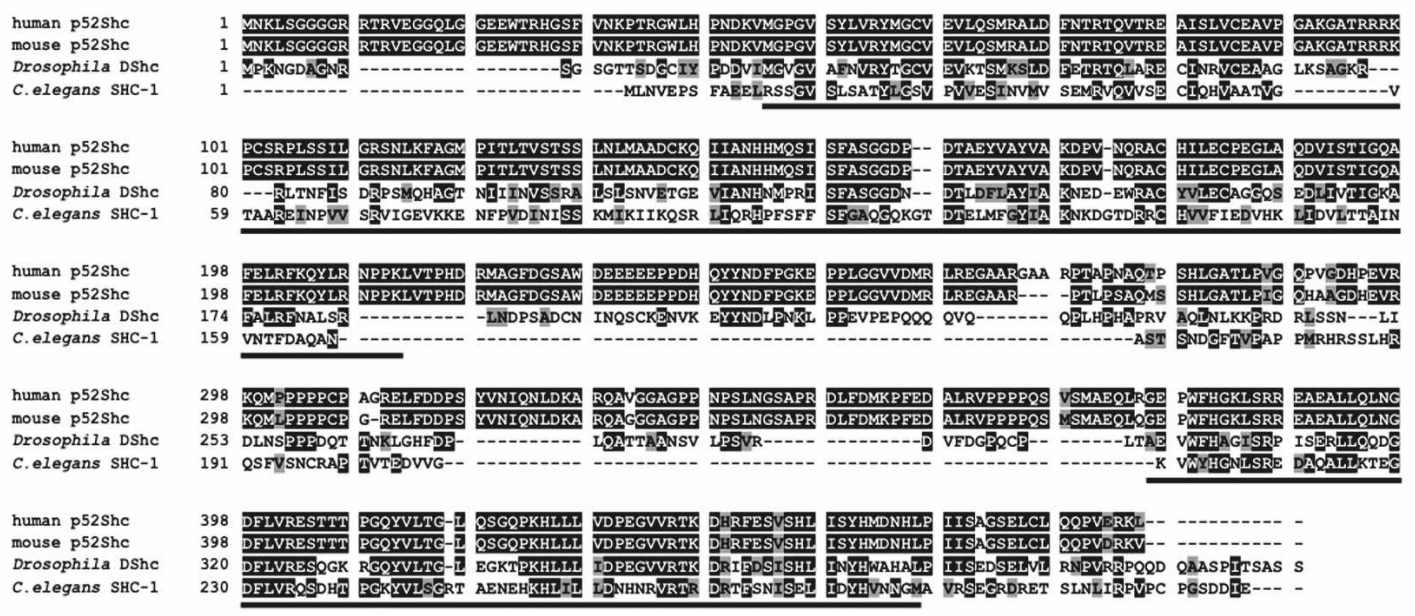

B

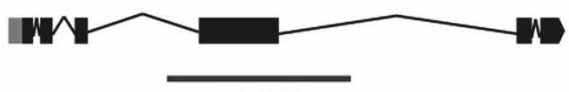

ok198

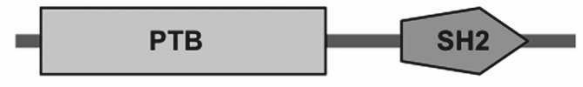

C
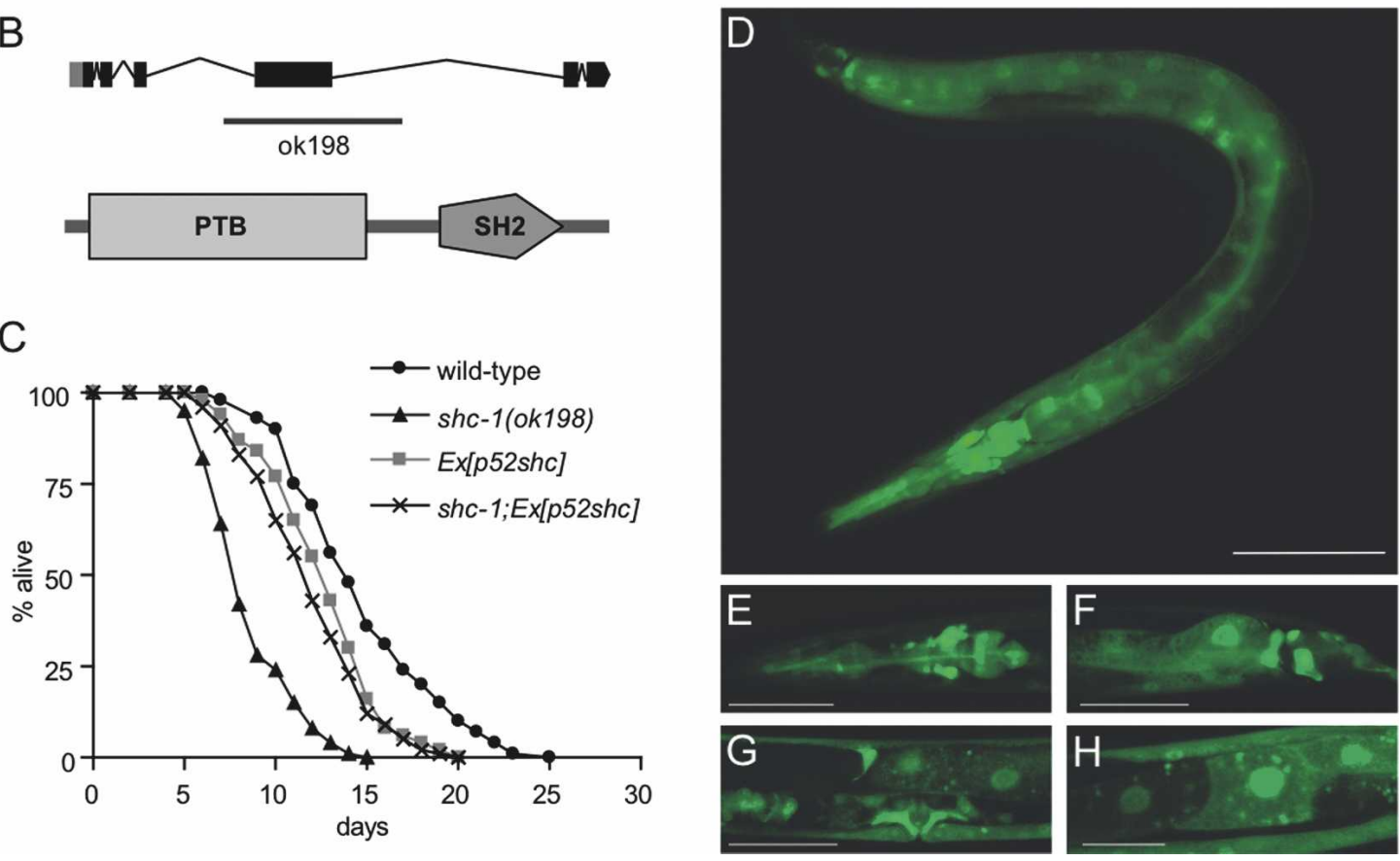

Figure 1. Characterization of shc-1. (A) Alignments of C. elegans SHC-1, human p52Shc, mouse p52Shc, and Drosophila DShc. Identical amino acid residues are highlighted, and similar residues are shaded. The conserved PTB and SH2 domains are underlined. (B) Genomic organization of shc-1 and location of deletion in ok198. Coding regions are indicated by boxes, and introns are represented as lines. SHC-1 has the N- to C-terminal PTB-SH2 modularity of Shc-like proteins (predicted by SMART, Simple Modular Architecture Research Tool, http://smart.embl-heidelberg.de). (C) Life-span analysis of shc-1(ok198) and rescue by transgenic expression of human p52shc. All life-span data presented in this and subsequent figures are the result of at least three representative experiments. All life-span data are listed as mean life span \pm standard error of the mean; $(n)$ number of animals observed; (Ex) extrachromosomal array; $P$-values refer to experimental strain and wild-type control animals. shc-1(ok198): $8.9 \pm 0.1 \mathrm{~d}(n=152, P<0.0001)$; wild type: $14.4 \pm 0.1$ $\mathrm{d}(n=204) ;$ shc-1(ok198);Ex[p52Shc]: $12.0 \pm 0.4 \mathrm{~d}(n=93, P=0.1161$ vs. Ex[p52Shc]); Ex[p52shc]: $12.7 \pm 0.3 \mathrm{~d}(n=82, P<0.0001) .(D-H)$ shc-1::gfp is broadly expressed in C. elegans. SHC-1::GFP stains the pharynx, the majority of head neurons $(E)$, the tail neurons $(F)$, the vulva muscles, and the gonads $(G)$. $(H)$ SHC-1::GFP localizes to both the cytoplasm and nuclei of intestinal cells. A portion of SHC-1 is localized at the cytoplasmic membrane. The image in $D$ was taken with a $20 \times$ objective on a Zeiss Axioplan2 microscope using an EGFP filter set (480/20-nm excitation, 510/20-nm emission). To estimate the autofluorescent background signal, we imaged wild-type worms under the same conditions and detected only a faint yellow fluorescence signal in intestinal cells. Bar, $100 \mu M$. $(E-H)$ Higher-magnification pictures were taken on a confocal laser microscope, always showing L4 larvae. Bars: $E-G, 50 \mu M ; H, 25 \mu M$.

shown). This deletion removes part of the PTB and eliminates translation of the SH2 domain. Therefore, shc- 1(ok198) likely represents a strong loss-of-function or null allele (Fig. 1B). 
In mammals, p66Shc has been implicated in life-span regulation. This prompted us to examine whether shc-1 affects aging in C. elegans. shc-1(ok198) mutants have a substantially reduced mean adult life span of $8.9 \pm 0.1 \mathrm{~d}$ compared with $14.4 \pm 0.1 \mathrm{~d}$ in wild type (Fig. 1C). To explore the functional relationship between $s h c-1$ and human p52Shc, we asked whether p52Shc can rescue the loss of shc-1 in C. elegans. Therefore, we expressed human p52Shc in C. elegans under control of the shc-1 promoter used for the GFP expression studies described above. Using two independent transgenic lines, we found that human p52Shc efficiently rescued the short-lifespan phenotype of shc-1(ok198) mutants (Fig. 1C). This clearly indicates a functional complementarity between shc-1 and human p52Shc in life-span regulation and suggests that shc-1 and p52Shc are orthologs.

Loss of shc-1 function results in accelerated aging and increased stress sensitivity

shc-1(ok198) mutants display a strongly reduced mean adult life span (38\% compared with wild type). To further explore the role of SHC-1 in life-span regulation, we tested whether loss of $s h c-1$ function results in any obvious morphological or developmental defect that might account for the reduced life span. shc-1(ok198) animals appeared morphologically normal, reproduced normally (brood size $322 \pm 8[n=10]$ compared with $348 \pm 12$ $[n=10]$ in wild type), and had a normal generation time $(2.8 \pm 0.07 \mathrm{~d}[n=50]$ compared with $2.8 \pm 0.04 \mathrm{~d}[n=31]$ in wild type). However, shc-1(ok198) animals displayed an egg-laying (Egl) defect: $46 \%$ of the animals died prematurely and showed a "bag of worms" phenotype, re- sulting from progeny hatching inside the uterus of the mother. To rule out the possibility that life-span modulation directed by shc-1 is merely the consequence of developmental abnormalities and defective egg-laying, we censored animals that became Egl during the lifespan analysis.

To alleviate problems due to premature death caused by egg-laying defects, typically either the drug 2 '-deoxy5-fluorouridine (FUDR) that prevents growth of progeny is administered or life span is tested in the fer-15 mutant background. fer-15(b26) mutants do not produce progeny due to defective spermatogenesis, but life span remains unaffected (Friedman and Johnson 1988). Since in several of our previous experiments the antimetabolite FUDR affected life span in an unpredictable way (W. Qi and R. Baumeister, unpubl.), we crossed shc-1 with fer-15 mutants to avoid egg-laying defects. Life span in the shc1;fer-15 double mutant was significantly shorter than in fer-15 alone (Supplemental Table 1). We conclude that the accelerated aging of shc-1(ok198) mutants is not simply the cause of defective egg-laying.

We next asked whether the life-span regulation by shc-1 is gene dose-dependent. To overexpress shc-1, we constructed transgenic worms containing the entire genomic region of $s h c-1$ fused to the $3-\mathrm{kb}$ upstream regulatory sequence. Transgenic expression of shc-1 from an incompletely penetrant, extrachromosomal array rescued the short-life-span phenotype of the shc-1 mutant. shc-1(ok198);Ex[shc-1::gfp] showed a $42 \%$ life-span extension compared with shc-1(ok198) and displayed a mean lifetime of $12.6 \pm 0.4 \mathrm{~d}$ (Fig. $2 \mathrm{~A})$. However, wildtype animals carrying the shc-1::gfp transgene did not show extension of life span relative to wild type. This
Figure 2. Loss of shc-1 function results in accelerated aging and increased stress sensitivity. (A) Life-span analysis of shc-1(ok198) and rescue by transgenic expression of shc$1:: g f p$. shc-1(ok198): $8.9 \pm 0.1$ d $(n=152$, $P<0.0001)$; wild type: $14.4 \pm 0.1 \mathrm{~d}(n=204)$; Ex[shc-1::gfp]: $13.6 \pm 0.5 \mathrm{~d}(n=96, P=0.582)$; shc-1(ok198); Ex/shc-1::gfp]: $12.6 \pm 0.4 \mathrm{~d}$ $(n=106, P=0.0229$ vs. Ex[shc-1::gfp]). (B) shc-1(ok198) displays an increase of gut lipofuscin autofluorescence as compared with similarly aged wild-type animals at $23^{\circ} \mathrm{C}$. Quantification of the wild-type and shc1(ok198) populations' gut autofluorescence ( $n=20$ per strain) at days 0,4 , and 8 of adulthood. Error bars represent the standard error of the mean. $\left({ }^{\star \star}\right) \quad P<0.0001 .(C)$ shc1 (ok198) is sensitive to heat stress $\left(32^{\circ} \mathrm{C}\right)$. $n=40$ per each strain and time point. $(D)$ shc1(ok198) is sensitive to oxidative stress (100 $\mathrm{mM}$ paraquat) compared with wild-type. $n=100$ per each strain.
A

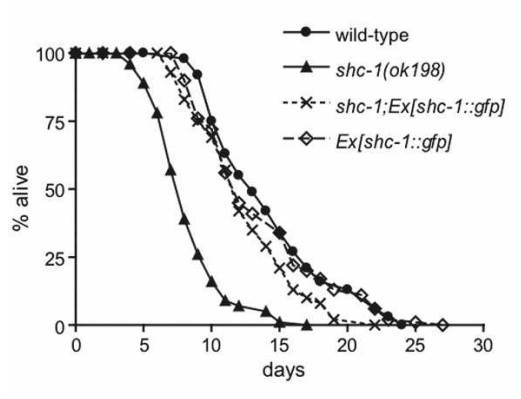

C

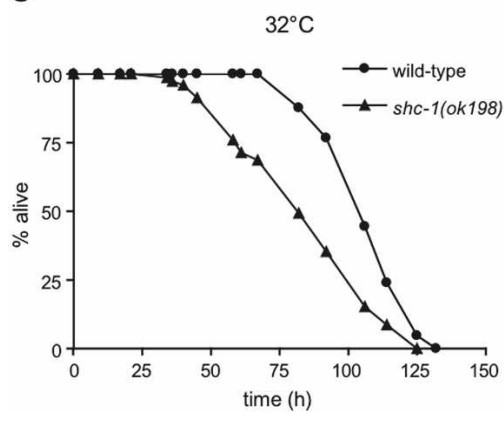

B

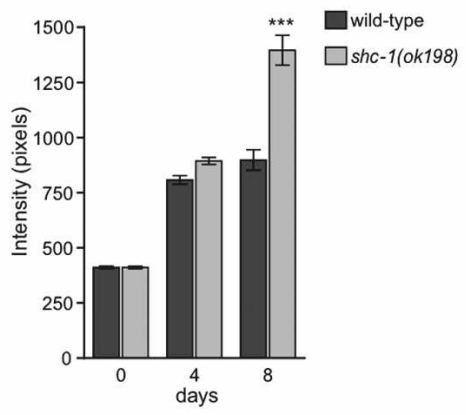

D

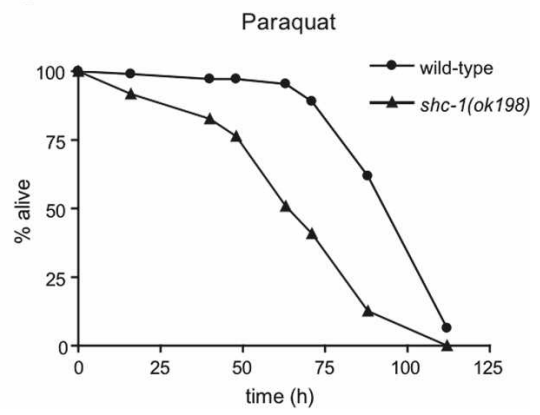


result demonstrates that the deletion in the shc-1 gene in shc-1(ok198) indeed is responsible for the mutant phenotype, since it can be complemented by the shc-1 transgene.

To further support the hypothesis that the short life span of shc-1(ok198) mutants is due to accelerated aging rather than due to a more general pathology, we monitored the accumulation of intestinal autofluorescence in adult animals. Intestinal autofluorescence, which is caused by lysosomal deposits of lipofuscin, accumulates over time in the aging animal and is an established marker for aging (Garigan et al. 2002). Consistently with its short life span, the shc-1 mutant accumulated intestinal autofluorescence more rapidly than the wild type (Fig. 2B). The average level of autofluorescence in shc1(ok198) increased with age in a statistically significant manner $(P<0.0001)$. Accordingly, loss of shc-1 function caused progressive decline in pharyngeal pumping rate (Supplemental Fig. 1A) and body movement (Supplemental Fig. 1B) compared with wild type, two well-described behavioral changes in aging worms (Huang et al. 2004). Furthermore, shc-1 mutants showed many morphological features of aging worms like flaccid appearance, tissue deterioration, and necrotic cavities at earlier age (Supplemental Figure 1C; Garigan et al. 2002; Chow et al. 2006). Together, we conclude that mutations in shc-1 result in a premature aging phenotype.

C. elegans mutants that display life-span phenotypes typically also show altered responses to stress, including oxidative and heat stress (Finkel and Holbrook 2000; Lithgow and Walker 2002). These findings are consistent with the theory that aging may be caused by cumulative cellular and systemic damage involving ROS. To test this correlation, we examined the survival of shc1(ok198) mutants exposed to oxidative and heat stress. In accordance with its life-span phenotype, we found that shc-1(ok198) exhibited a significant, up to $32 \%$ (after $67 \mathrm{~h}$ at $32^{\circ} \mathrm{C}$ ) stronger sensitivity to heat stress than wild type (Fig. 2C). We also analyzed sensitivity to oxidative stress. We exposed animals to paraquat, a generator of superoxide anions, and found that shc-1 mutants were substantially more sensitive (up to $44 \%$ after $63 \mathrm{~h}$ ) than wild type (Fig. 2D).

\section{shc-1 modulates IIS upstream of daf-16}

In C. elegans, the IIS pathway is a prominent regulator of both life span and stress response. The FOXO transcription factor DAF-16 is essential for the increased life span and stress resistance of $d a f-2$ and other IIS mutants (Ogg et al. 1997). First, we asked if life span of shc-1 mutants depends on daf-16. If daf-16 was the predominant effector of $s h c-1$ life-span modulation, then daf-16 overexpression should suppress shc-1, and loss of daf-16 function should not have an additional effect on the aging phenotype of $s h c-1$ mutants. To test this, we analyzed the effect of daf-16(RNAi) on shc-1(ok198) life span. Animals treated with daf-16(RNAi) indeed lived as long as shc-1 mutants, and daf-16(RNAi) did not further reduce shc-1-shortened life span (Fig. 3A). Furthermore, we tested the effect of two daf-16-null alleles on shc-1 life span: Neither daf-16(mgDf50) nor daf-16(mu86) increased the short life span of shc-1 mutants (Fig. 3B; Supplemental Table 1). These experiments were performed in an fer-15 mutant background to prevent progeny. Interestingly, the hypomorphic daf-16(m26) allele partially suppressed the life-span reduction of shc1(ok198) (Supplemental Table 1). Overexpression of daf16 extended the life span of shc-1 mutant animals. However, the animals carrying integrated daf-16::gfp did not live as long as wild-type animals (Fig. 3C), suggesting that either $d a f-16:: g f p$ on its own results in a phenotype (this has been suggested before: Henderson and Johnson 2001) or SHC-1 may have additional effects on other lifespan-promoting factors that have to be identified (see below). From these findings, we hypothesized that shc-1 and daf-16 may affect life span by a shared mechanism.

As daf-16 is essential for oxidative stress response, we also tested whether $d a f-16$ contributes to the increased stress sensitivity of shc-1 mutants. We found that mutating daf-16 did not further enhance the sensitivity of shc-1 to oxidative stress. The daf-16 shc-1 double mutant exhibited sensitivity to paraquat similar to shc-1 and daf-16 single mutants (Fig. 3F). Together, our data suggest that daf-16 is required for the shc-1(ok198)-mediated aging and stress phenotype and indicate that shc-1 acts upstream of daf-16.

\section{shc- 1 acts upstream of age- 1 and daf-2}

IIS is primarily mediated through the PI3-kinase AGE-1. As mammalian Shc acts as an adaptor molecule in receptor tyrosine kinase signaling, we wondered whether SHC-1 might function through AGE-1 in C. elegans. We reasoned that, if SHC-1 exerts its effects upstream of AGE-1, then loss of age-1 function should suppress the shc-1 aging phenotype. To test this hypothesis, we created shc-1;age-1 double mutants and determined their life span. Remarkably, age-1(hx546) completely suppressed the shortened life span of shc-1(ok198) (Fig. 3D) as the double mutants had a mean life span similar to that of the long-lived age-1 mutants. This indicates that age-1 is epistatic to shc-1 and positions age-1 downstream from shc-1. The placement of shc-1 upstream of age-1 in the IIS pathway was also corroborated by oxidative stress experiments. age-1 mutants, similar to mutants in a variety of components of the IIS pathway, confer increased resistance to oxidative stress (Yanase et al. 2002). We found that the high sensitivity of shc-1(ok198) to the oxidative stressor paraquat was fully suppressed by age-1(hx546) (Fig. 3F). In conclusion, these genetic results demonstrate that shc-1 may be positioned upstream of age-1 in the IIS pathway for the control of life span and stress response.

Activation of the insulin receptor in human cells results in the interaction of Grb2-SOS with IRS-1, PI3K, and Shc (Skolnik et al. 1993). Since the C terminus of DAF-2 contains an extension that may function analogously to mammalian IRS-1, we wondered whether shc-1 exerts its effects through the insulin-like receptor DAF- 
Neumann-Haefelin et al.

Figure 3. shc-1 acts in the IIS pathway for the control of life span and stress response. (A) daf-16(RNAi) does not reduce shc-1 mutant life span. Wild type on pL4440: $15.4 \pm 0.4 \mathrm{~d} \quad(n=99) ; \quad s h c-1$ (ok198) on pL4440: $10.8 \pm 0.3 \mathrm{~d}(n=90, P<0.0001)$; daf-16(RNAi): $\quad 10.1 \pm 0.2$ d $\quad(n=100$, $P<0.0001$ ); shc-1(ok198); daf-16(RNAi): $10.1 \pm 0.3 \mathrm{~d}[n=82, P=0.6713$ vs. daf16(RNAi)]. (B) Life-span analysis of daf16(mgDf50) and shc-1(ok198). The assays were performed in a fer-15(b26) background at $25^{\circ} \mathrm{C}$ to prevent progeny. shc1(ok198); fer-15(b26): $10.5 \pm 0.2 \mathrm{~d}(n=160$, $P<0.0001)$; fer-15(b26): $14.0 \pm 0.2 \mathrm{~d}(n=160)$; daf-16(mgDf50); fer-15(b26): $10.1 \pm 0.2 \mathrm{~d}$ ( $n=107, P<0.0001)$; daf-16(mgDf50) shc1(ok198); fer-15(b26): $10.2 \pm 0.3 \mathrm{~d}$ ( $n=104$, $P<0.0001) .(C)$ Overexpression of daf-16 partially suppresses the $s h c-1$ mutant life span. shc-1(ok198): $8.8 \pm 0.3 \mathrm{~d}(n=70)$; wild type: $14.4 \pm 0.1 \mathrm{~d}(n=204)$; Is [daf$16:: g f p): 15.8 \pm 0.5 \mathrm{~d}(n=139, P=0.0437)$; shc-1(ok198); Is[daf-16::gfp]: $11.1 \pm 0.4 \mathrm{~d}$ ( $n=90, P<0.0001)$. (D) age-1(hx546) suppresses the shc-1(ok198) aging phenotype. Wild type: $14.2 \pm 0.4 \mathrm{~d}(n=204) ;$ shc- 1 (ok198): $8.4 \pm 0.3 \mathrm{~d}(n=152, P<0.0001)$; shc-1(ok198); age-1(hx546): $17.5 \pm 0.5 \mathrm{~d}$ ( $n=178, P<0.0001) ;$ age-1(hx546): $17.6 \pm 0.5$ $\mathrm{d}(n=245, P<0.0001)$. (E) daf-2(e1368) extends the short life span of shc-1(ok198). Wild type: $14.3 \pm 0.35 \mathrm{~d}(n=104) ;$ shc-1 (ok198): $10.6 \pm 0.3 \mathrm{~d}(n=103, P<0.0001)$; daf-2(e1368): $25.3 \pm 0.6$ d $\quad(n=110$, $P<0.0001) ; \quad$ shc-1(ok198); daf-2(e1368): $20.2 \pm 0.6 \mathrm{~d}(n=109, P<0.0001)$. (F) Effect of daf-2(e1370), daf-16(m26), and age1(hx546) on the oxidative stress sensitivity of shc-1(ok198). Animals were exposed to $200 \mathrm{mM}$ and $100 \mathrm{mM}$ paraquat, respectively. All data are listed as mean survival \pm standard error of the mean, $n=100$ per each strain. Data were combined from at least two independent experiments. (G) daf-2(e1370) increases the life span of shc-1(ok198). The assay was performed in a fer-15(b26) background at $25^{\circ} \mathrm{C}$ to prevent progeny. fer-15(b26): $13.0 \pm 0.2 \mathrm{~d}(n=217)$; shc-1(ok198); fer-15(b26): $10.7 \pm 0.2 \mathrm{~d}(n=216, P<0.0001$ vs. fer-15); fer-15(b26); daf-2(e1370): $31.8 \pm 0.9 \mathrm{~d}(n=160, P<0.0001$ vs. fer-15); shc-1(ok198); fer-15(b26); daf-2(e1370): $27.2 \pm 0.6 \mathrm{~d}(n=210$, $P<0.0001$ vs. fer-15). (H) The accelerated rate of lipofuscin accumulation in shc-1(ok198) is suppressed by daf-2(e1370). Quantification of the gut autofluorescence of fer-15(b26), shc-1(ok198); fer-15(b26), fer-15(b26); daf-2(e1370), and shc-1(ok198); fer-15(b26); daf$2(e 1370)$ at day 10. Error bars represent the standard error of the mean. $\left(^{\star \star \star}\right) P<0.0001$ comparing shc-1 with the shc-1; daf-2 mutant. (I) SHC-1 interacts with DAF-2 in HEK 293T cells. Membrane-bound sIg7-tagged DAF-2 (sIg.7.DAF-2) or control proteins (sIg7-tag and sIg7.control protein) were coexpressed with Flag-tagged SHC-1 (F.SHC-1) in HEK 293T cells and precipitated with Protein G Sepharose. (Top) Western blot analysis was performed with anti-Flag antibody. (Bottom) Expression levels of all proteins in the lysates and precipitates are shown.

2. To test this, we constructed shc-1;daf-2 double mutants and analyzed their life span. Mutations that compromise the activity of daf-2 extend life span, and this longevity is dependent on functional daf-16. The short life span of shc-1(ok198) was greatly increased by daf2(e1368) (Fig. 3E). To further explore the possibility that shc-1 might function through daf-2, we tested the interaction with a different, stronger daf- 2 allele. At $25^{\circ} \mathrm{C}$, daf-2(e1370) significantly extended the shortened life span of shc-1(ok198) (Fig. 3G). This genetic interaction between shc-1 and daf-2 was further corroborated by oxidative stress experiments. Here we found that the high 
sensitivity of shc-1(ok198) to paraquat was completely suppressed by daf-2(e1370), which confers increased tolerance to oxidative stress (Fig. 3F). From these observations, we suppose that shc-1 modulates life span and stress response through the DAF-2 insulin-like receptor of the IIS pathway. In the absence of viable daf-2 deficiency mutants, a precise relationship between shc-1 and daf-2 is difficult to determine, but the complete suppression of shc-1 stress sensitivity by daf-2 mutation strongly supports that shc-1 acts genetically upstream of daf-2. Although epistasis analyses using nonnull mutants need to be interpreted with great caution, the lack of full suppression of shc-1 life span by daf-2 could indicate the presence of an additional pathway downstream from shc-1, but in parallel to DAF-2 (see also below).

Next we asked whether the long-lived daf-2 mutation could also suppress the premature accumulation of the aging marker lipofuscin in shc-1(ok198). The rate of gut autofluorescence was dramatically decreased in shc1(ok198) when combined with the daf-2(e1370) mutation, which correlates well with the ability of daf2(e1370) to suppress the short life span of shc-1 mutant animals (Fig. $3 \mathrm{H}$; Supplemental Fig. S2). The rate of autofluorescence in the shc-1;daf-2 double mutant was similar to that observed in the daf-2 mutant, corroborating our model of shc-1 being upstream of daf-2.

To determine whether SHC-1 physically contacts the DAF-2 receptor, we tested the biochemical interaction in human embryonic kidney (HEK) 293T cells. These cells were transiently cotransfected with plasmids coding for Flag-tagged C. elegans SHC-1 and a membrane-bound fusion (sIg7) of the cytoplasmic domain of DAF-2. We immunoprecipitated sIg7-tagged DAF-2 from cell extracts and, as demonstrated in Figure 3I, coprecipitated SHC-1 specifically with DAF-2, but not with the controls (sIg7 or an unrelated protein). We conclude that SHC-1 can directly interact with DAF-2 in vitro. SHC-1 is an adaptor protein that contains an $\mathrm{N}$-terminal PTB domain followed by a C-terminal SH2 domain. To map the DAF-2 protein interaction sites, we generated truncations of SHC-1. Both the PTB domain and the SH2 domain coprecipitated with DAF-2 from HEK 293T cell lysates (Supplemental Fig. 3), indicating that both domains have the capability to bind DAF-2, but the interaction with the PTB domain seems to be stronger. Together, our data suggest that SHC-1 genetically and physically interacts with the DAF- 2 receptor and support a model in which SHC-1 may act upstream of DAF-2 to modulate IIS-dependent stress response and aging in $C$. elegans.

\section{shc-1 function promotes nuclear translocation} of $D A F-16$

The FOXO transcription factor DAF-16 is the major downstream target of C. elegans IIS. Reduction of IIS activity, as well as response to stress, promotes DAF-16 nuclear localization, where it functions as a transcriptional regulator (Lin et al. 2001).

We showed above that the premature aging and the high stress sensitivity observed in shc-1(ok198) depends on active DAF-16. Therefore, we wondered whether mutant shc-1 might affect the nuclear accumulation of DAF-16. To test this, we examined DAF-16 intracellular localization in shc-1 mutant and wild-type animals after heat-shocking a transgenic DAF-16::GFP strain, and distinguished three phenotypic categories: cytosolic, nuclear, or intermediate localization of the DAF$16::$ GFP fusion protein. In wild-type animals, DAF$16::$ GFP is predominantly localized to intestinal nuclei after heat shock $198 \%$ nuclear localization in Is [daf$16:: g f p] ; n=93$ ) (Fig. 4). In the shc-1(ok198) mutant, the number of animals with nuclear localization of GFP signal was decreased to $42 \%(n=99)$ (Fig. 4), whereas the cytosolic localization increased accordingly. This finding suggests that wild-type shc-1 facilitates nuclear translocation of the FOXO transcription factor DAF-16 upon induction of heat stress.

\section{shc-1 is involved in the JNK signaling pathway}

Based on genetic studies, a role for Shc in Ras-dependent MAPK activation has been established in mammals. The JNK family, a subgroup of the MAPK superfamily, is part of a signal transduction cascade that is activated upon exposure to environmental stress. In C. elegans, the JNK pathway comprises the JNK ortholog JNK-1 and two MKK4/7 orthologs, MEK-1 and JKK-1. Disruption of JNK signaling components in C. elegans resulted in accelerated aging and hypersensitivity to oxidative and thermal stress (Oh et al. 2005). Interestingly, the life-span reduction of jnk-1 mutants fully depends on the transcription factor DAF-16. Therefore, the most likely explanation is that the insulin and JNK pathways act in parallel to converge on DAF-16 (Oh et al. 2005).

To investigate a possible link between SHC-1 and the JNK pathway, we assayed the life span and stress response of animals deficient for SHC-1 and JNK-1. The jnk-1(gk7) mutant animals showed a shorter life span compared with wild type, and knockdown of jnk-1 did not further decrease the life span of short-lived shc-1 animals. The shc-1;jnk-1 double mutant displayed a mean life span similar to ink-1(gk7) (Fig. 5A). Furthermore, expression of an additional, genomically inserted copy of jnk-1 in the shc-1(ok198) mutant significantly increased life span (Fig. 5B). We hypothesized that shc-1 depends on ink-1 function, and their effects on aging might be mediated by a shared mechanism. The genetic interaction between shc-1 and jnk-1 was further supported by oxidative stress experiments. We found that the high sensitivity of shc-1(ok198) to oxidative stress was not enhanced by jnk-1(gk7) (Fig. 6A). In summary, these results suggest that $s h c-1$ has a dual role and affects life span and stress response by interfering with both the JNK and the IIS pathways.

\section{shc-1 acts upstream of mek-1 in the JNK pathway}

Activation of JNK-1 by its upstream kinases is critical for its function. Two JNK-kinases, MEK-1 and JKK-1, 
A

DAF-16::GFP Localization in Intestinal Cells

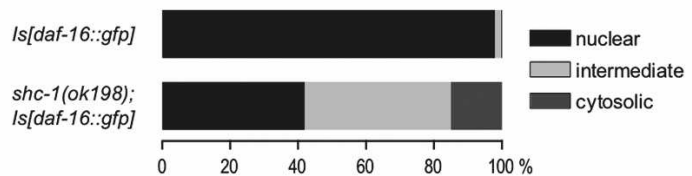

B
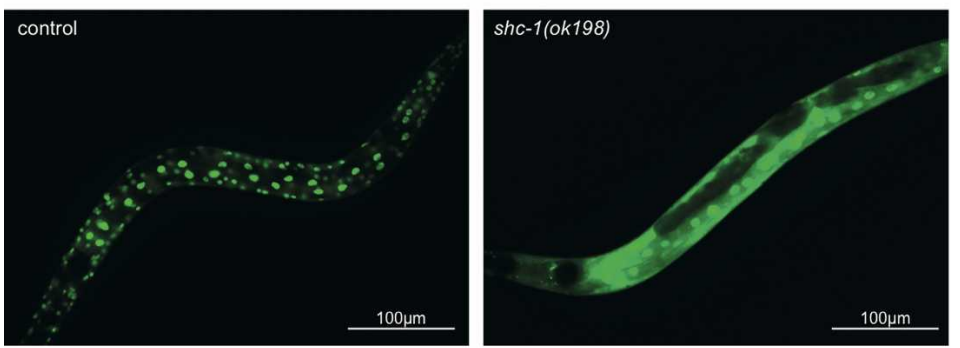

Figure 4. Inhibiting $s h c-1$ function reduces DAF-16 nuclear localization in the intestinal cells. $(A)$ Quantification of DAF-16 intestinal nuclear and cytosolic localization after 90-min heat shock in Is [daf-16::gfp] (control animals, $n=93$ ) and shc-1(ok198); Is (daf$16:: g f p]$ animals $(n=99)$. The graph represents multiple experiments. shc-1(ok198) reduces the number of animals with intestinal DAF-16 nuclear localization, $P<0.0001$. (B) Images of intestinal cells from control animals Is[daf-16::gfp] and shc-1(ok198); Is [daf$16:: g f p]$ animals displaying DAF-16 nuclear localization. Images were taken at $200 \times$ and $630 \times$ magnification.
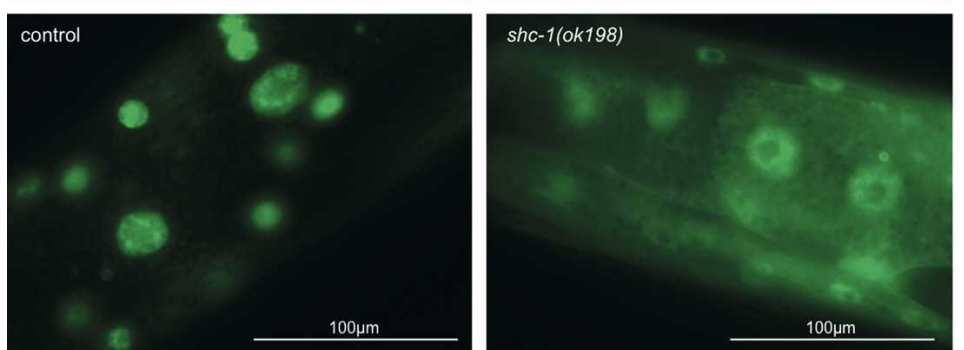

have been described in C. elegans. First, we tested whether shc-1 regulates the JNK pathway by modulating JKK-1. The $j k k-1(\mathrm{~km} 2)$-null mutants have a short life span compared with wild type, and $j k k-1$ further reduced the already short life span of shc-1(ok198). The shc$1 ; j k k-1$ double mutant displayed a shorter life span than each single mutant (Fig. 5C). Furthermore, transgenic expression of $j k k-1$ modestly extended the short life span of the shc-1 mutant (Fig. 5D). These data suggest an additive effect of $s h c-1$ and $j k k-1$ on life-span regulation. However, we found that $j k k-1(k m 2)$ exhibited normal sensitivity to paraquat, and the shc-1;jkk-1 double mutant behaved similarly as shc-1(ok198) (Fig. 6A). From these data, a precise relationship between shc-1 and $j k k-1$ could not be determined, but their additive effect on life span suggests that shc-1 might act independently of $j k k-1$.

In addition to JKK-1, the C. elegans genome encodes another JNK kinase called MEK-1. If shc-1 exerts its effects on JNK signaling by regulating mek-1, then the mek-1(ks54) mutant phenotype should overwrite any effect of shc-1(ok198). To test this hypothesis, we first analyzed the life span of mek-1(ks54) and also created shc-1;mek-1 double mutants. Surprisingly, we here observed that mek-1(ks54) mutants have a short life span, whereas it was reported previously that this mutant has normal life span (Oh et al. 2005). We had extensively backcrossed the mutation prior to testing, suggesting that the differences between these two studies may have been caused by distinct genetic backgrounds. The double mutant had a short life span that was indistinguishable from that of each single mutant (Fig. 5E). Independent confirmation of a downstream function of mek-1 in the shared pathway came from overexpression of mek-1 that completely suppressed the reduced life span of the shc-1 mutant (Fig. 5F). Furthermore, we also found that the shc-1;mek-1 double mutant displayed a strong sensitivity to oxidative stress similar to that of the mek-1 mutant (Fig. 6A), indicating that oxidative stress sensitivity in shc-1 mutants depends on mek-1 function. These observations support a model in which shc-1 modulates life span and stress response through the mek-1-mediated branch of the JNK pathway, positioning shc-1 upstream of mek-1.

MEK-1 has also been implicated in the response to heavy metal stress. Heavy metals play an important role in the generation of ROS, and exposure to heavy metals can induce stress-related physiology associated with aging. To determine whether SHC-1 is involved in the response to heavy metal stress, we examined the effect of shc-1 mutation on copper sensitivity in the mek-1(ks54) background. Similar to the mek-1(ks54) mutation, we found that the shc-1 mutation caused a strong sensitivity to $\mathrm{CuSO}_{4}$. Wild-type animals grew well and reached adulthood within $4 \mathrm{~d}$ on plates containing $40 \mu \mathrm{M} \mathrm{CuSO}_{4}$, while shc-1(ok198) and mek-1(ks54) animals grew only poorly. Only $38 \%$ of the $s h c-1$ worms reached adulthood within the same period of $4 \mathrm{~d}$. The shc-1;mek-1 double mutant exhibited a similar enhanced sensitivity to $\mathrm{CuSO}_{4}$ than the mek-1 single mutant (Fig. 6B), indicating that $s h c-1$ controls heavy metal stress response together with mek-1. 
A
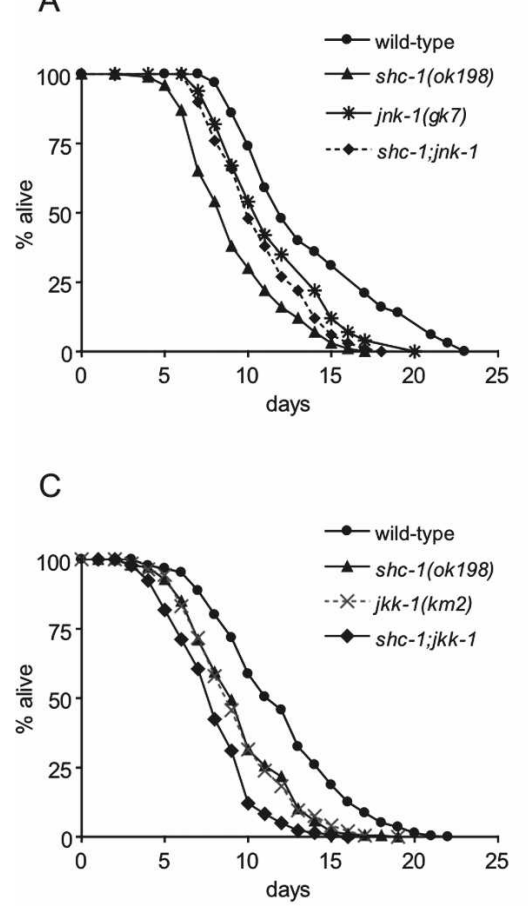

E

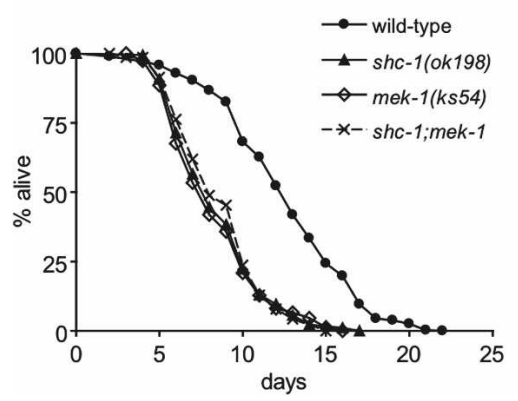

B

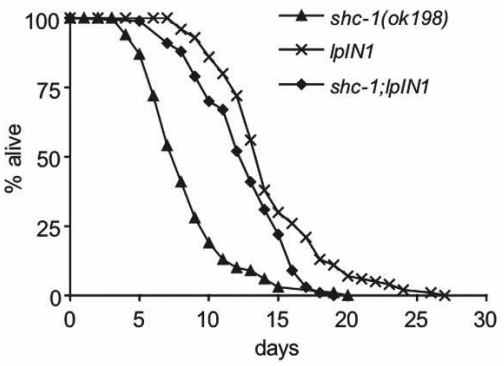

D

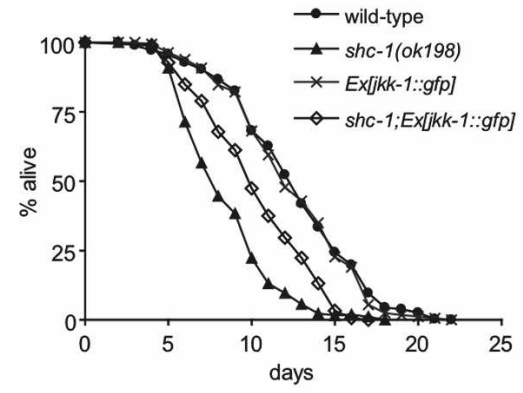

$\mathrm{F}$

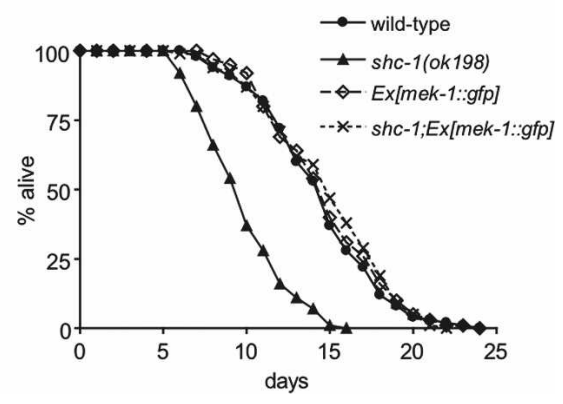

Figure 5. shc-1 acts in the JNK signaling pathway for the control of life span. (A) ink1 (gk) suppresses the shc-1(ok198) aging phenotype. Wild type: $14.2 \pm 0.4 \mathrm{~d}(n=204)$; shc1(ok198): $8.4 \pm 0.3 \mathrm{~d}(n=152, \quad P<0.0001)$; jnk-1(gk7): $11.6 \pm 0.2 \mathrm{~d}(n=320, P<0.0001)$; shc-1(ok198);jnk-1(gk7): $10.9 \pm 0.1 \mathrm{~d}(n=232$, $P<0.0001)$. (B) Transgenic overexpression of jnk-1 suppresses shc-1(ok198). shc-1(ok198): $8.4 \pm 0.3 \mathrm{~d}(n=152, P<0.0001) ;$ shc-1;IpIn1: $12.5 \pm 0.3 \mathrm{~d}(n=116, \quad P<0.0001) ; \quad \operatorname{lpIn} 1:$ $14.5 \pm 0.3 \mathrm{~d}(n=123, P=0.708) .(C)$ shc- 1 and $j k k-1$ act independently to control life span. Wild type: $12.0 \pm 0.2 \mathrm{~d} \quad(n=277) ;$ shc1(ok198): $9.5 \pm 0.2 \mathrm{~d}(n=207, \quad P<0.0001)$; $j k k-1(\mathrm{~km} 2): 9.5 \pm 0.2 \mathrm{~d}(n=197, P<0.0001)$; shc-1(ok198); jkk-1(km2): $8.1 \pm 0.2 \mathrm{~d}(n=132$, $P<0.0001)$. (D) Life-span analysis of shc1 (ok198) and transgenic strains expressing $j k k-1$. Wild type: $12.7 \pm 0.2 \mathrm{~d}(n=308)$; shc1(ok198): $8.6 \pm 0.2 \mathrm{~d}(n=175, P<0.0001) ; E X^{-}$ [jkk-1::gfp]: $12.5 \pm 0.3 \mathrm{~d}(n=163, P=0.595)$; shc-1(ok198);Ex[jkk-1::gfp]: $\quad 10.4 \pm 0.3 \quad \mathrm{~d}$ $(n=152, P<0.0001)$. (E) Life-span analysis of shc-1(ok198) and mek-1(ks54). Wild type: $12.7 \pm 0.2 \mathrm{~d}(n=308) ;$ shc-1(ok198): $8.6 \pm 0.2 \mathrm{~d}$ ( $n=175, P<0.0001) ;$ mek-1(ks54): $8.4 \pm 0.2 \mathrm{~d}$ (n =148, $\quad P<0.0001) ; \quad$ shc-1(ok198); mek1(ks54): $8.7 \pm 0.2 \mathrm{~d}(n=139, P<0.0001) .(F)$ Transgenic overexpression of mek-1 suppresses shc-1 mutants. Wild type: $14.5 \pm 0.3 \mathrm{~d}$ $(n=109)$; shc-1(ok198): $9.9 \pm 0.3 \mathrm{~d}(n=100$, $P<0.0001) ; \quad$ Ex[mek-1::gfp]: $14.9 \pm 0.3 \mathrm{~d}$ $(n=118, \quad P=0.467) ; \quad$ shc-1(ok198);Ex[mek$1:: g f p]: 14.9 \pm 0.4 \mathrm{~d}(n=104, P=0.454)$.
As an adaptor protein, a potential role of SHC-1 might be to bind proteins in the JNK-1 pathway. For this purpose, we tested whether SHC-1 and the JNK kinase MEK-1 directly interact with each other. We observed that Flag-tagged SHC-1 coprecipitated with MEK-1, but not with a control protein, from transfected HEK 293T cells (Fig. 6C). To validate this result under in vivo conditions, we performed coimmunoprecipitation experiments from whole-worm lysates, using C. elegans strains expressing transgenic MEK-1::GFP and Flagtagged SHC-1 (Fig. 6D). Indeed, we found that SHC-1 coprecipitates with MEK-1::GFP, confirming that this interaction occurs in vivo. Therefore, SHC-1 mediates its role in the JNK-1 pathway by direct interaction with MEK-1, a kinase that acts upstream of JNK-1.

To investigate which domain of SHC-1 mediates the association with MEK-1, we generated SHC-1 truncations and coexpressed these constructs together with MEK-1 in HEK 293T cells. We found that MEK-1 specifically coprecipitates with the SH2 domain of SHC-1 (Supplemental Fig. 4) but not with the PTB domain. This observation suggests that SHC-1 might function as a scaffold protein and form protein complexes with DAF-2 and MEK-1. Our results also indicate that different protein domains of SHC-1 interact with DAF-2 and MEK-1, respectively.

JNK signaling and IIS act in parallel to converge on DAF-16. We conclude that, in contrast to its inhibitory effects on IIS, shc-1 positively regulates JNK signaling. To further support this hypothesis, we analyzed DAF16::GFP localization at conditions of reduced IIS. Knockdown of daf-2 function by RNAi causes DAF-16 translocation to the nucleus. However, in the shc-1 mutant, DAF-16 is less efficiently delivered to the nucleus after daf-2(RNAi) (Supplemental Fig. 5). This finding suggests that SHC-1, via JNK-1, is also required for the nuclear localization of DAF-16 in the absence of IIS.

\section{Discussion}

A substantial body of evidence has demonstrated the importance of p52/46Shc in Ras/MAPK activation and 
Figure 6. SHC-1 acts upstream of MEK-1 to control oxidative and heavy metal stress response, and directly interacts with MEK-1. $(A)$ shc-1, mek-1, and jnk-1 act together in oxidative stress response. jnk-1(gk7), shc-1(ok198), and double mutants display similar sensitivity to paraquat. mek-1(ks54) contributes to shc-1(ok198) oxidative stress sensitivity. A representative experiment is shown in which animals had been exposed to $100 \mathrm{mM}$ paraquat. Please note the different time points for survival analysis. All data are listed as mean survival \pm standard error of the mean, $n=100$ per each strain. (B) shc1(ok198) and mek-1(ks54) are sensitive to $\mathrm{CuSO}_{4}$. The number of adults was counted after $4 \mathrm{~d}$ of incubation at $20^{\circ} \mathrm{C}$. All data are listed as mean numbers of adults \pm standard deviation of the mean, $n=400$ per each strain. The graphs presented are the result of at least three representative trials. (C) SHC-1 interacts with MEK-1 in HEK 293T cells. V5tagged MEK-1 (V5.MEK-1) or a control protein (V5-NPHP1) was coexpressed with Flagtagged SHC-1 (F.SHC-1) in HEK 293T cells and precipitated with anti-V5 antibody. (Top) Western blot analysis was performed with anti-Flag antibody. (Bottom) Expression levels of all proteins in the lysates are shown. $(D)$ MEK-1::GFP and Flag-tagged SHC-1 (F.SHC1) coimmunoprecipitate from whole-worm lysates. Total lysates were prepared from transgenic worms that express F.SHC-1 and MEK-1::GFP or GFP as control and subjected to immunoprecipitation with anti-GFP antibody. Precipitates were probed by immunoblotting with anti-Flag (top) and anti-GFP (bottom). The expression level of F.SHC-1 in worm lysates is shown.
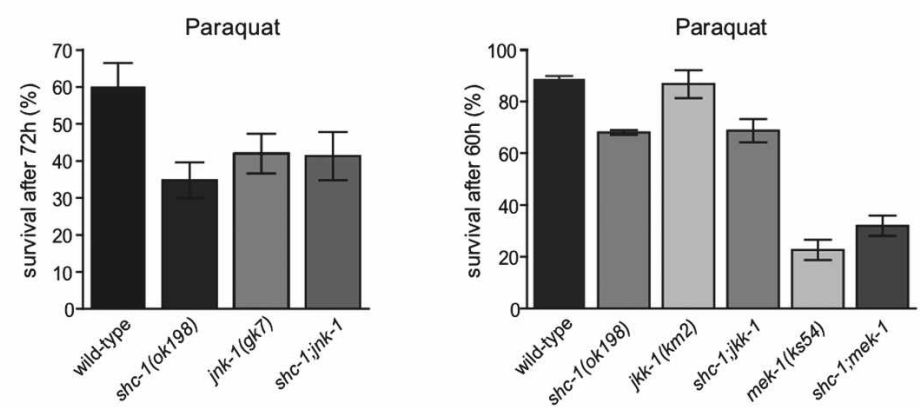

B

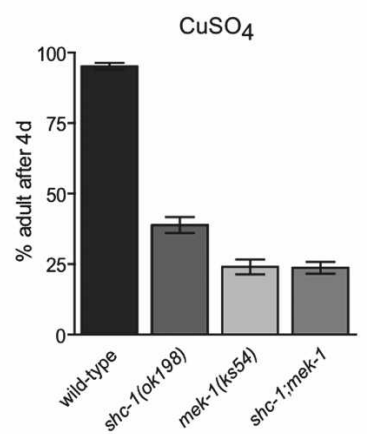

C

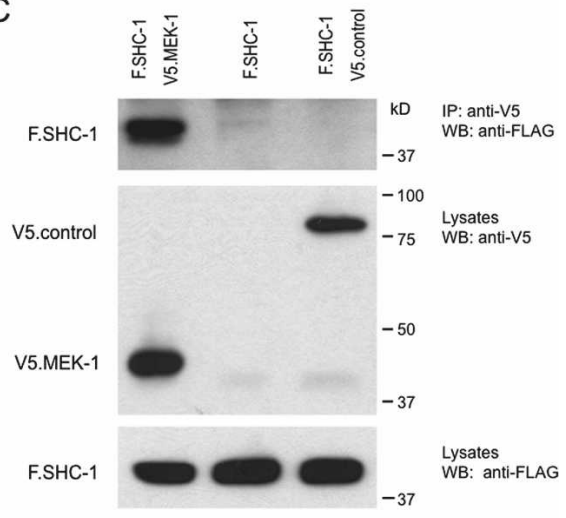

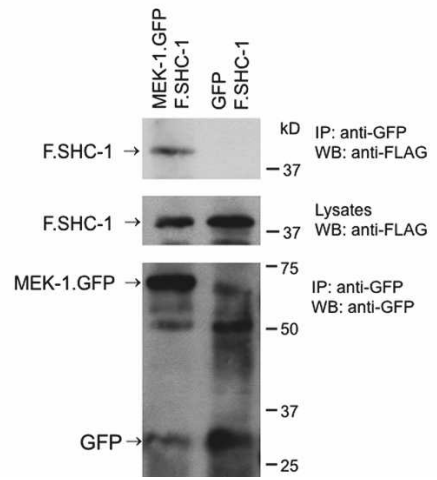

regulation of developmental and growth processes. So far, only p66Shc has been implicated in the regulation of life span in mammals. In this study, we provide evidence that the C. elegans homolog of p52Shc, SHC-1, acts as a modulator of life span and stress response. Our data reveal that $s h c-1$ plays a dual role as a negative regulator of IIS signaling and as an activator of the JNK signaling pathway. Remarkably, both aspects involved in the regulation of stress response and longevity converge on controlling the activation of the FOXO transcription factor DAF-16.

We demonstrate here that shc-1(ok198) mutant animals show a substantially reduced life span that is accompanied by enhanced sensitivity to heat, oxidation, and heavy metal stress. Although it is generally difficult to interpret the cause of reduced life span, several findings argue that shc-1 mutant animals, indeed, age pre- maturely and do not simply die from a general pathology. First, shc-1 mutants accumulate intestinal autofluorescence more rapidly than wild type and develop typical signs of aging worms at earlier age. Second, down-regulation of genes encoding factors in the IIS pathway effectively suppress the early death of shc-1 animals. Overexpression of shc-1 alone is not sufficient to extend life span beyond that level reached by wild type, which is compatible with the notion that SHC-1, as an adaptor protein, requires the presence of interacting factors to be effective.

Several genetic pathways that regulate C. elegans life span have been shown to also influence life span in higher organisms (for review, see Kenyon 2005). Interestingly, C. elegans shc-1 has opposite effects from the mammalian p66Shc knockout. Mice lacking p66Shc are long-lived and resistant to oxidative stress. The p66 iso- 
form has a unique function in mammals, and this effect has entirely been attributed to the additional $\mathrm{N}$-terminal $\mathrm{CH} 2$ domain present in the p66 isoform, which functions as a stress sensor (Migliaccio et al. 1999; Gertz et al. 2008) and is absent in p52/46Shc. In contrast, knockout of p52/46Shc isoforms in mice results in embryonic lethality at day 11.5 (Lai and Pawson 2000). The developmental lethality currently precludes the analysis of p52/46Shc functions later in life. A p66Shc ortholog does not exist in C. elegans. However, the fact that shc-1 mutant animals were rescued by the expression of human p52Shc, together with the high degree of sequence similarity of both proteins, suggest that shc-1 resembles the worm ortholog of p52Shc. Thus, it will be interesting to see whether p52Shc in mammals might have a similar, post-embryonic role in stress response and longevity.

In C. elegans as in higher vertebrates, IIS is one of the most important pathways for the regulation of stress response and life span. Our data strongly support that SHC-1 contributes to the control of life span and stress response by modulating IIS upstream of the PI3 kinase AGE-1 and the insulin/IGF receptor DAF-2. Both, mutations in age-1 and daf-2 effectively suppressed the short life span and the oxidative stress sensitivity of shc-1 mutants, suggesting that shc-1 is genetically positioned upstream of age-1 and daf-2 in the IIS. Moreover, daf-2(e1370) suppressed the premature accumulation of lipofuscin in shc-1 mutant animals.

Since our coimmunoprecipitation studies revealed that SHC-1 physically interacts with DAF-2 in vitro and both domains of SHC-1, the SH2 and preferably the PTB were capable of binding DAF-2, the most likely explanation is that SHC-1 functions as an adaptor/modulator of DAF-2. Such a role is further supported by the localization of a subpopulation of SHC-1::GFP in close proximity to the cytoplasmic membrane.

How SHC-1 negatively regulates DAF-2 signaling needs to be determined in future experiments. SHC-1 might function to control either the kinetics or extent of DAF-2 activation. For example, SHC-1 could help to recruit a kinase to the DAF-2 receptor that modulates DAF-2 activity by phosphorylation. Our preliminary data, in fact, suggest that, upon expression in cell culture, coexpression of SHC-1 results in a strong increase of DAF-2 phosphorylation (our unpublished data). Another explanation might be that SHC-1 regulates DAF-2 receptor internalization; indeed, the association of p52Shc with the endocytic machinery has been reported in mammalian cells (Okabayashi et al. 1996). Alternatively, the inhibitory effect of SHC-1 might be more indirect; for example, through removing the AGE-1 complex from a source of PIP2, the precursor of PIP3 (Haugh and Meyer 2002).

In addition to its inhibitory role in the IIS pathway, $s h c-1$ also contributes to JNK signaling. We placed $s h c-1$ upstream of $j n k-1$, since $j n k-1$ overexpression could suppress the shc-1 mutant phenotype. Together with the similarity of the shc-1 and jnk-1 mutant phenotypes, this suggests that SHC-1 positively affects JNK-1 function. JNK-1 signaling in C. elegans is activated by two
JNK kinases that act in parallel, MEK-1 and JKK-1. Our data support a role for shc-1 in the regulation of mek-1, but not of $j k k-1$. First, shc-1(ok198) further accelerates $j k k-1$ aging, suggesting an additive effect. Second, like ink-1, overexpression of mek-1 rescued shc-1 short life span. Third, SHC-1 and MEK-1 were shown to directly interact with one another by coimmunoprecipitation from HEK293T cells. Moreover, this interaction can occur in vivo as we demonstrated by immunoprecipitation from whole-worm protein lysates. An interaction of both proteins has previously been demonstrated by yeast twohybrid methods in the C. elegans Interactome Project (Li et al. 2004). Our data are indicative of SHC-1 having distinct interaction surfaces for DAF-2 and MEK-1 binding.

mek-1 plays a pivotal role in stress response to heavy metals (Koga et al. 2000; Mizuno et al. 2004). Interestingly, we found that shc-1 is also involved in the heavy metal stress response. shc-1 and mek-1, as well as shc1;mek-1 double mutants, are similarly hypersensitive to copper ions, indicating that both shc-1 and mek-1 act in the same pathway to control response to heavy metal stress. However, according to our own as well as previously reported data, $j n k-1$ and $j k k-1$ are not involved in heavy metal response (Mizuno et al. 2004; our unpublished data). This might suggest that the pathway controlling oxidative stress response/life span and heavy metal response branch downstream from SHC-1 and MEK-1.

An important issue concerns the mechanism by which SHC-1 binding to MEK-1 activates signaling through the JNK cascade. We found that only the SH2 domain of SHC-1 binds efficiently to MEK-1. Activation of MEK-1 might thus involve transient association with the SH2 domain; the PTB domain might then mediate the translocation of this complex to the plasma membrane, where MEK-1 could interact with MAPKK kinases or small GTPases like Ras. Indeed, the localization of the PTB domain to membranes has been observed in mammalian cells before (Lotti et al. 1996). Thus, Shc proteins might function as a molecular scaffold to assemble MAPK signaling modules. Whether both interactions with DAF-2 and MEK-1 occur synchronously, establishing an efficient cross-talk between IIS and JNK-1 signaling, is an interesting question that needs to be targeted by future research.

Both the effects of life-span reduction and stress sensitivity caused by the shc-1(ok198) mutation converge on daf-16. Our data strongly support this hypothesis since neither two different daf-16 deficiency alleles nor daf-16(RNAi) increased the life-span reduction of shc1(ok198). We obtained corresponding results from oxidative stress experiments. Thus, we suggest that SHC-1 affects DAF-16 activity in dual ways. First, by inactivating the IIS kinases cascade, SHC-1 contributes to reducing DAF-16 phosphorylation, causing its nuclear translocation. Indeed, our results show that in a shc-1 mutant, the amount of nuclearly localized DAF-16::GFP is strongly reduced upon heat stress. Second, SHC-1 facilitates, via JNK-1, DAF-16 nuclear localization and probably also its activation. Therefore, we assume that, in shc-1(ok198) animals, the remaining nuclear fraction of DAF-16 might not be fully active due to the reduced 
JNK-1 signaling (Fig. 7). Reduced JNK-1 signaling diminishes DAF-16 nuclear phosphorylation and, therefore, its activity as a transcription factor. This second aspect probably cannot be compensated simply by elevating expression of transgenic DAF-16, since the latter only becomes activated through JNK-1-mediated phosphorylation that in turn requires active SHC-1. Consistent with such a model, overexpression of daf-16 could only partially rescue the $s h c-1$ phenotype, and the hypomorphic daf-16(m26) mutation (which expresses a mutant DAF16 protein) even slightly extended shc-1 life span. daf16(m26) carries a splice donor site mutation in intron 2 that specifically affects the daf-16a transcripts (Lee et al. 2001). One possible explanation could be that $d a f-16 b$ transcripts, in the absence of $d a f-16 a$, retain some degree of DAF-16 activity even in the background of defective JNK-1 signaling, or that $d a f-16 b$ expression in the somatic gonad and pharynx is not affected in a shc1(ok198) mutant. Alternative models are possible, and could, for example, involve a negative feedback loop through which daf-16 affects shc-1 activity (our unpublished results). Additional studies will be required to solve this issue.

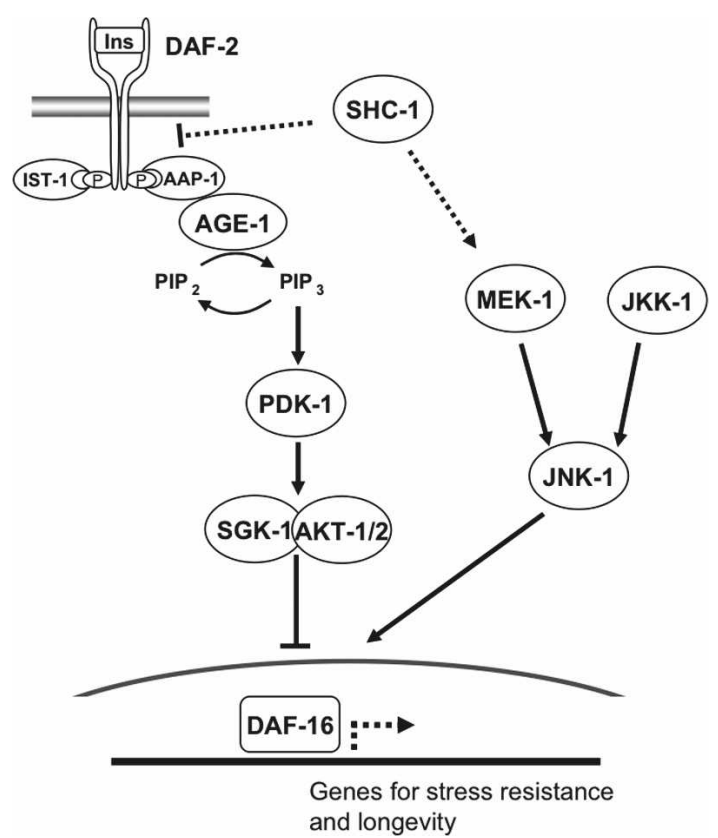

Figure 7. Model for the function of SHC-1 in IIS and JNK signaling. Upon DAF-2 activation, AGE-1 and PIP3 activate PDK-1 and the downstream kinase complex AKT-1/AKT-2/SGK-1. The transcription factor DAF-16 is directly inhibited and phosphorylated via activity of the IIS pathway, a function of which is induction of stress response and longevity genes. In addition, DAF-16 integrates positive signals via JNK signaling stimulating nuclear accumulation. Genetic and biochemical analyses presented here suggest that SHC-1 opposes IIS upstream of AGE-1 and DAF-2 and directly interacts with DAF-2. Furthermore, SHC-1 activates JNK signaling and interacts with MEK-1 to control life span and response against heat, oxidation, and heavy metals.
Interestingly, we observed that DAF-16 accumulation in intestinal nuclei is reduced in shc-1 mutants after daf-2(RNAi), whereas DAF-16 in wild-type animals treated with daf-2(RNAi) is exclusively nuclear. This finding suggests that the efficient translocation of DAF16 to the nucleus after blocking IIS requires SHC-1/ JNK-1 activity.

Collectively, our data suggest a model in which the adaptor protein SHC-1/p52Shc is a modulator of life span and stress response. SHC-1 depresses IIS by binding to DAF-2, the C. elegans insulin/IGF receptor, and activates JNK signaling via MEK-1 interaction (Fig. 7). Both pathways converge on the FOXO transcription factor DAF-16, and shc-1-mediated effects on IIS and JNK signaling eventually help to control both the nuclear translocation and activation of DAF-16. SHC-1 may, thus, serve to coordinate cellular responses to changes in the environment and help to prevent senescence. The identification of this endocrine function of shc-1 supports and extends its proposed role in MAPK signaling by amplifying and coordinating stress responses.

\section{Materials and methods}

\section{Strains}

The strains used were as follows: wild-type N2 Bristol, NH3119 shc-1(ok198) (outcrossed four times), DR26 daf-16(m26), BR3449 daf-16(m26) shc-1(ok198), DH26 fer-15(b26), BR3699 shc-1(ok198); fer-15(b26), BR4949 daf-16(mu86); fer-15(b26), BR4948 daf-16(mu86) shc-1(ok198); fer-15(b26), BR4950 daf16(mgDf50); fer-15(b26), BR4951 daf-16(mgDf50) shc-1(ok198); fer-15(b26), DR1572 daf-2(e1368), BR4633 shc-1(ok198); daf2(e1368), BR4631 fer-15(b26); daf-2(e1370), BR4693 shc1(ok198); fer-15(b26); daf-2(e1370), TJ1052 age-1(hx546), BR3592 shc-1(ok198); age-1(hx546), TJ356 Is [daf-16::gfp], BR3999 shc-1(ok198); Is[daf-16::gfp], DR2278 aap-1(m889), BR3695 shc-1(ok198) aap-1(m889), VC8 ink-1(gk7) (additionally outcrossed four times to our N2), BR3740 shc-1(ok198); jnk-1(gk7), KU2 jkk-1(km2), BR3761 shc-1(ok198); jkk-1(km2), FK171 mek-1(ks54) (additionally outcrossed two times to our N2), BR3929 shc-1(ok198); mek-1(ks54), lpIn1[jnk-1; pRF4] (Oh et al. 2005), BR3530 shc-1(ok198); IpIn1, BR3876 Ex[jkk-1::gfp; pRF4], BR3952 shc-1(ok198); Ex[jkk-1::gfp; pRF4], BR3421 Ex[shc-1::gfp; pRF4], BR3423 Ex[shc-1::gfp; pRF4], BR3446 shc1(ok198); Ex[shc-1::gfp; pRF4], BR3447 shc-1(ok198); Ex[shc$1:: g f p ; p R F 4]$, BR3925 Ex[humanp52shc::gfp; pRF4], BR3926 Ex[human p52shc::gfp; pRF4], BR3964 shc-1(ok198); Ex[human p52shc::gfp; pRF4], BR4221 shc-1(ok198); Ex[human p52shc::gfp; pRF4], BR4248 Ex[mek-1::gfp], BR4318 shc1(ok198); Ex[mek-1::gfp], BR5090 Ex/mek-1::gfp; Pshc1::Flag::shc-1; Pmyo-2::dsRed], BR5091 Ex[mek-1::gfp; Pshc1::Flag::shc-1; Pmyo-2::dsRed], BR5094 Ex[Pmek-1::gfp; Pshc-1::Flag::shc-1; Pmyo-2::dsRed].

\section{Plasmids}

We generated an shc-1::gfp translational fusion (pBY2361) by inserting an SphI/BamHI fragment containing the complete genomic shc-1 locus (predicted gene F54A5.3a) and 3-kb upstream regulatory sequence of $s h c-1$ into pPD95.75 (A. Fire, Carnegie Institute of Washington, Baltimore, MD). To express human p52Shc in C. elegans, 3-kb upstream regulatory sequences of shc-1 were inserted at the SphI/NheI sites and fused to human p52Shc cDNA, which was inserted at the NheI/XbaI sites of 
pPD95.75 (pBY2375). To generate mek-1::gfp (pBY2359), a 5.5$\mathrm{kb}$ fragment containing the complete genomic mek-1 locus and 4-kb upstream regulatory sequences were inserted at the SphI/ XmaI sites of pPD95.75.

To express Flag-tagged shc-1 in C. elegans, shc-1 cDNA was amplified and fused to the Flag epitope by PCR. The $3-\mathrm{kb}$ promoter region of shc-1 was inserted at the SphI/NheI sites and fused to Flag-tagged shc-1, which was inserted at the NheI/XmaI sites of pPD95.75 (pBY2792). To create Pmek-1::gfp, the 4-kb promoter region of mek-1 was cloned into GFP vector pPD95.75 (pBY2791).

For expression in cell culture, $\mathrm{N}$-terminally Flag-tagged SHC-1 (pBY2387) was constructed by amplifying shc-1 cDNA and cloned into a modified pcDNA6 vector (Benzing et al. 2001). Flag-tagged truncations of SHC-1 containing the PTB domain (corresponding to residues 1-167) or SH2 domain (residues 209316) were generated by subcloning the respective cDNA into pcDNA6. To create N-terminally tagged MEK-1, C. elegans MEK-1 cDNA was cloned into pcDNA3.1/nV5-Dest (Invitrogen) using the GATEWAY cloning technology. A membranebound fusion of the C-terminal cytoplasmic domain of DAF-2 (sIg.7.DAF-2) was generated using a derivate of pcDNA6 containing the leader sequence of CD5 followed by the $\mathrm{CH} 2$ and $\mathrm{CH} 3$ domains of human IgG1 and the transmembrane region of CD7 (Tsiokas et al. 1997). The cytoplasmic part of DAF-2 was inserted directly $\mathrm{C}$-terminal of the $\mathrm{CD} 7$ transmembrane region. V5-tagged NPHP1 and Flag-tagged CD2AP controls were provided by G. Walz and sIg7-tagged Celsr2 was provided by $\mathrm{T}$. Schäfer (University Hospital, Freiburg, Germany).

\section{Transgenic strains}

To generate animals expressing shc-1::gfp, plasmid pBY2361 was injected at $10 \mathrm{ng} / \mathrm{\mu L}$ into N2, along with rol-6 (pRF4, 90 $\mathrm{ng} / \mu \mathrm{L}$ ) as coinjection marker (Mello et al. 1991). Two independent transgenic lines (BR3421, BR 3423) were analyzed for the expression pattern of shc-1, crossed into NH3119 shc-1(ok198), and used for life-span assays. For rescue experiments of shc1(ok198) with human p52Shc, plasmid pBY2375 was injected at $30 \mathrm{ng} / \mu \mathrm{L}$ together with $70 \mathrm{ng} / \mathrm{\mu L}$ pRF4 into N2. Two independent transgenic lines (BR3925, BR3926) were then crossed into NH3119 shc-1(ok198) and analyzed for life span. To generate a jkk-1 overexpressing strain (BR3876), pMK104 (Kawasaki et al. 1999) was injected at $30 \mathrm{ng} / \mu \mathrm{L}$ together with $80 \mathrm{ng} / \mu \mathrm{L} \mathrm{pRF} 4$. A mek-1 overexpressing strain (BR4248) was generated by injecting pBY2359 at $5 \mathrm{ng} / \mathrm{\mu L}$ together with $70 \mathrm{ng} / \mathrm{\mu L}$ pRF4. For coimmunoprecipitation analysis from $C$. elegans, transgenic strains expressing mek-1::gfp and Flag::shc-1 were created (BR5090 and BR5091). Plasmid pBY2359 was injected at $5 \mathrm{ng} / \mathrm{\mu L}$ together with pBY2792 $(5 \mathrm{ng} / \mu \mathrm{L})$ and myo-2::dsRed $(20 \mathrm{ng} / \mu \mathrm{L})$ in N2. As control, a GFP-expressing and Flag::shc-1-expressing strain (BR5094) was generated by injecting pBY2791 (5 ng/ $\mathrm{LL})$,

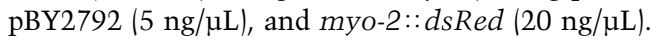

\section{Life-span analysis}

Life-span assays were conducted in general as described in Lakowski and Hekimi (1998). All assays were performed at $23^{\circ} \mathrm{C}$, unless otherwise noted. At this temperature, animals are not subjected to the mild stress conditions observed at $25^{\circ} \mathrm{C}$, but, in addition, temperature-sensitive mutants already showed a strong phenotype that would not be easily detectable at $20^{\circ} \mathrm{C}$. For RNAi life-span experiments, nematodes were placed on NGM plates containing $1 \mathrm{mM}$ isopropyl- $\beta$-D-thiogalactopyranoside (IPTG) and seeded with HT115(DE3) bacteria transformed with either the pL4440 vector or the test RNAi construct. Synchronized worms were raised at $15^{\circ} \mathrm{C}$ to the $\mathrm{L} 4$ larval stage, then transferred to fresh plates and shifted to $23^{\circ} \mathrm{C}$.
The late L4 larval stage was counted as day 0. Fertile strains were transferred every other day to fresh plates until progeny production ceased. Animals were examined every day for touchprovoked movement until death. Animals that crawled off the plate, displayed extruded internal organs, or displayed an Egl phenotype were censored. All life-span assays were repeated at least three times. GraphPad Prism 4.0 software (GraphPad Software Inc.) was used to calculate mean adult life span and to perform statistical analysis. $P$-values were determined using a log-rank test (Mantel-Cox).

\section{Stress assays}

Heat and oxidative stress assays were performed as described in Hertweck et al. (2004). For heat stress assays $\left(32^{\circ} \mathrm{C}\right)$, 40 young adult worms per each time point and each strain were checked for viability. Survival was scored by gently prodding each animal with a platinum wire. For oxidative stress assays, $100 \mathrm{mM}$ paraquat (N,N'-dimethyl-4-4'-bipyridinium dichloride) was used. For each strain, $5 \times 15$ young adult worms were tested at $20^{\circ} \mathrm{C}$ and counted after $3 \mathrm{~d}$ for viability. For metal stress assays, worms were grown on normal NGM plates before being tested on $\mathrm{CuSO}_{4}$-enriched plates containing $40 \mu \mathrm{M} \mathrm{CuSO}_{4}$ and seeded with Escherichia coli OP50. Three worms were allowed to lay eggs for $2 \mathrm{~h}$, and the number of eggs was counted (a plate usually contained $\sim 20-30$ eggs). Per each strain, five plates were analyzed. The number of adults was counted after $4 \mathrm{~d}$ of incubation at $20^{\circ} \mathrm{C}$. All stress assays were repeated at least three times.

\section{Autofluorescence analysis}

Adult worms were grown at $23^{\circ} \mathrm{C}$ on NGM plates. Endogenous gut fluorescence was photographed using a Zeiss GFP filter set (450-490 nm excitation, 500-550 nm emission). Images were collected with a CCD camera using 12-bit digitalization and identical exposure times and beam pass settings at the same day. Autofluorescence was quantified using Axiovision 4.4 software (Zeiss) ( $n=10$ for each time point per strain). $P$-values were calculated using the unpaired $t$-test. The autofluorescence assay was repeated at least three times.

\section{Analysis of aging phenotypes}

Pharyngeal pumping assays were performed on NGM agar plates with OP50. Pumping was assessed by observing the number of pharyngeal contractions during a 10-sec interval $(n=10$ animals at each time point).

To analyze body bends, adult hermaphrodites were transferred in M9 on a Petri dish and the number of body bends per $10 \mathrm{sec}$ was counted ( $n=15$ animals at each time point). All assays were repeated three times.

\section{DAF-16 nuclear localization assay}

For DAF-16::GFP translocation studies, day 1 reproductive adult worms were placed on NGM plates seeded with OP50 at $35^{\circ} \mathrm{C}$. After 90 -min heat shock, worms were immediately mounted onto the slide with $5 \mathrm{mM}$ sodium azide in M9 buffer. We visualized the nuclear translocation of DAF-16::GFP with a Zeiss Axioplan2 microscope. We scored animals as having nuclear-localized DAF-16::GFP if the majority of intestinal cells displayed a distinct concentration of GFP in the nucleus. Other cell types were not evaluated. The translocation assay was repeated at least five times.

\section{Cell culture, transfection, and immunoprecipitation}

Coimmunoprecipitation experiments were performed as described (Huber et al. 2003). Briefly, HEK 293T was cultured in 
DMEM supplemented with $10 \%$ fetal bovine serum and transiently transfected by the calcium phosphate method. After incubation for $24 \mathrm{~h}$, cells were washed and lysed in a $1 \%$ Triton X-100 lysis buffer. Following ultracentrifugation $(100,000 g, 30$ $\min , 4^{\circ} \mathrm{C}$ ), the supernatant was incubated for $2 \mathrm{~h}$ at $4^{\circ} \mathrm{C}$ with appropriate antibody prebound to protein G-Sepharose beads (Amersham Pharmacia Biotech). Bound proteins were resolved by SDS-PAGE and analyzed by immunoblot. Antibodies were obtained from Sigma (anti-Flag M2 mAb), Serotec (anti-V5 $\mathrm{mAb}$ ), and GE healthcare (anti-human $\mathrm{Ab}$ ).

\section{Immunoprecipitation from whole-worm lysate}

Transgenic worms expressing Flag::SHC-1, MEK-1::GFP, or GFP as control were used for coimmunoprecipitation analysis. Mixed-stage worms were resuspended in $100 \mathrm{mM}$ Tris/ $\mathrm{HCl} / \mathrm{pH}$ $8.0), 200 \mathrm{mM} \mathrm{NaCl}, 1 \mathrm{mM}$ EDTA, $8 \%$ glycerol, and protease inhibitors, and homogenized in a stainless steel homogenizer. After centrifugation to remove cellular debris, the supernatant was subjected to an ultracentrifugation $(100,000 \mathrm{~g})$ for $30 \mathrm{~min}$, followed by preclearing with protein $\mathrm{A} / \mathrm{G}$ agarose beads. For immunoprecipitation, extracts were incubated with polyclonal anti-GFP antibody (Clontech) for $1 \mathrm{~h}$ followed by $1 \mathrm{~h}$ of incubation with protein A/G agarose beads. Beads were washed with IP buffer. Bound proteins were resolved by SDS-PAGE and analyzed by immunoblotting using anti-Flag antibody (Sigma) and anti-GFP antibody (anti-GFP B-2 mAb; Santa Cruz Biotechnologies).

\section{Acknowledgments}

We thank T. Stiernagle and the Caenorhabditis Genetics Center for worm strains, the C. elegans Gene Knockout Consortium for providing us with the deletion mutant shc-1(ok198), and Y. Kohara (Genome Biology Laboratory, National Institute of Genetics, Mishima, Japan) for cDNA clones. We are grateful to A. Oelmann and A. Schwierzok for excellent technical help. We thank M. Gödel, B. Hartleben, and B. Schermer for reagents and for support during the coimmunoprecipitation experiments. This work was supported by a training grant of the Medical School Freiburg to E.N.H., by the EC 6th Framework Network of Excellence LIFESPAN (LSHG-CT-2007-036894) to M.H. and R.B., and by the Deutsche Forschungsgemeinschaft (CRC746), FRISYS (\#03139219, the Freiburg Initiative in Systems Biology, a program of the German Federal Ministry in Research and Education), and BMBF NGFN2 to G.W. and R.B.

\section{References}

Arantes-Oliveira, N., Apfeld, J., Dillin, A., and Kenyon, C. 2002. Regulation of life-span by germ-line stem cells in Caenorhabditis elegans. Science 295: 502-505.

Benzing, T., Gerke, P., Hopker, K., Hildebrandt, F., Kim, E., and Walz, G. 2001. Nephrocystin interacts with Pyk2, p130(Cas), and tensin and triggers phosphorylation of Pyk2. Proc. Nat1. Acad. Sci. 98: 9784-9789.

Bluher, M., Kahn, B.B., and Kahn, C.R. 2003. Extended longevity in mice lacking the insulin receptor in adipose tissue. Science 299: 572-574.

Chow, D.K., Glenn, C.F., Johnston, J.L., Goldberg, I.G., and Wolkow, C.A. 2006. Sarcopenia in the Caenorhabditis elegans pharynx correlates with muscle contraction rate over lifespan. Exp. Gerontol. 41: 252-260.

Clancy, D.J., Gems, D., Harshman, L.G., Oldham, S., Stocker, H., Hafen, E., Leevers, S.J., and Partridge, L. 2001. Extension of life-span by loss of CHICO, a Drosophila insulin receptor substrate protein. Science 292: 104-106.

Davis, R.J. 2000. Signal transduction by the JNK group of MAP kinases. Cell 103: 239-252.

Finkel, T. and Holbrook, N.J. 2000. Oxidants, oxidative stress and the biology of ageing. Nature 408: 239-247.

Friedman, D.B. and Johnson, T.E. 1988. A mutation in the age-1 gene in Caenorhabditis elegans lengthens life and reduces hermaphrodite fertility. Genetics 118: 75-86.

Garigan, D., Hsu, A.L., Fraser, A.G., Kamath, R.S., Ahringer, J., and Kenyon, C. 2002. Genetic analysis of tissue aging in Caenorhabditis elegans: A role for heat-shock factor and bacterial proliferation. Genetics 161: 1101-1112.

Gertz, M., Fischer, F., Wolters, D., and Steegborn, C. 2008. Activation of the lifespan regulator p66Shc through reversible disulfide bond formation. Proc. Natl. Acad. Sci. 105: 57055709.

Giorgio, M., Migliaccio, E., Orsini, F., Paolucci, D., Moroni, M., Contursi, C., Pelliccia, G., Luzi, L., Minucci, S., Marcaccio, M., et al. 2005. Electron transfer between cytochrome $c$ and p $66^{\text {Shc }}$ generates reactive oxygen species that trigger mitochondrial apoptosis. Cell 122: 221-233.

Gottlieb, S. and Ruvkun, G. 1994. daf-2, daf-16 and daf-23: Genetically interacting genes controlling Dauer formation in Caenorhabditis elegans. Genetics 137: 107-120.

Haugh, J.M. and Meyer, T. 2002. Active EGF receptors have limited access to PtdIns $(4,5) \mathrm{P}(2)$ in endosomes: Implications for phospholipase C and PI 3-kinase signaling. J. Cell Sci. 115: 303-310.

Henderson, S.T. and Johnson, T.E. 2001. daf-16 integrates developmental and environmental inputs to mediate aging in the nematode Caenorhabditis elegans. Curr. Biol. 11: 19751980.

Hertweck, M., Gobel, C., and Baumeister, R. 2004. C. elegans SGK-1 is the critical component in the Akt/PKB kinase complex to control stress response and life span. Dev. Cell 6: $577-588$.

Houthoofd, K., Braeckman, B.P., Lenaerts, I., Brys, K., De Vreese, A., Van Eygen, S., and Vanfleteren, J.R. 2002. Axenic growth up-regulates mass-specific metabolic rate, stress resistance, and extends life span in Caenorhabditis elegans. Exp. Gerontol. 37: 1371-1378.

Hsin, H. and Kenyon, C. 1999. Signals from the reproductive system regulate the lifespan of C. elegans. Nature 399: 362366.

Huang, C., Xiong, C., and Kornfeld, K. 2004. Measurements of age-related changes of physiological processes that predict lifespan of Caenorhabditis elegans. Proc. Natl. Acad. Sci. 101: 8084-8089.

Huber, T.B., Hartleben, B., Kim, J., Schmidts, M., Schermer, B., Keil, A., Egger, L., Lecha, R.L., Borner, C., Pavenstadt, H., et al. 2003. Nephrin and CD2AP associate with phosphoinositide 3-OH kinase and stimulate AKT-dependent signaling. Mol. Cell. Biol. 23: 4917-4928.

Kawasaki, M., Hisamoto, N., Iino, Y., Yamamoto, M., Ninomiya-Tsuji, J., and Matsumoto, K. 1999. A Caenorhabditis elegans JNK signal transduction pathway regulates coordinated movement via type-D GABAergic motor neurons. EMBO I. 18: 3604-3615.

Kenyon, C. 2005. The plasticity of aging: Insights from longlived mutants. Cell 120: 449-460.

Kenyon, C., Chang, J., Gensch, E., Rudner, A., and Tabtiang, R. 1993. A C. elegans mutant that lives twice as long as wild type. Nature 366: 461-464.

Kim, D.H., Liberati, N.T., Mizuno, T., Inoue, H., Hisamoto, N., Matsumoto, K., and Ausubel, F.M. 2004. Integration of Caenorhabditis elegans MAPK pathways mediating immunity 
and stress resistance by MEK-1 MAPK kinase and VHP-1 MAPK phosphatase. Proc. Natl. Acad. Sci. 101: 1099010994.

Koga, M., Zwaal, R., Guan, K.L., Avery, L., and Ohshima, Y. 2000. A Caenorhabditis elegans MAP kinase kinase, MEK-1, is involved in stress responses. EMBO J. 19: 5148-5156.

Lai, K.M. and Pawson, T. 2000. The ShcA phosphotyrosine docking protein sensitizes cardiovascular signaling in the mouse embryo. Genes \& Dev. 14: 1132-1145.

Lakowski, B. and Hekimi, S. 1998. The genetics of caloric restriction in Caenorhabditis elegans. Proc. Natl. Acad. Sci. 95: 13091-13096.

Lee, R.Y., Hench, J., and Ruvkun, G. 2001. Regulation of $C$. elegans DAF-16 and its human ortholog FKHRL1 by the daf-2 insulin-like signaling pathway. Curr. Biol. 11: 19501957.

Lee, S.S., Lee, R.Y., Fraser, A.G., Kamath, R.S., Ahringer, J., and Ruvkun, G. 2003. A systematic RNAi screen identifies a critical role for mitochondria in C. elegans longevity. Nat. Genet. 33: 40-48.

Li, S., Armstrong, C.M., Bertin, N., Ge, H., Milstein, S., Boxem, M., Vidalain, P.O., Han, J.D., Chesneau, A., Hao, T., et al. 2004. A map of the interactome network of the metazoan $C$. elegans. Science 303: 540-543.

Lin, K., Dorman, J.B., Rodan, A., and Kenyon, C. 1997. daf-16: An HNF-3/forkhead family member that can function to double the life-span of Caenorhabditis elegans. Science 278: 1319-1322.

Lin, S.J., Defossez, P.A., and Guarente, L. 2000. Requirement of NAD and SIR2 for life-span extension by calorie restriction in Saccharomyces cerevisiae. Science 289: 2126-2128.

Lin, K., Hsin, H., Libina, N., and Kenyon, C. 2001. Regulation of the Caenorhabditis elegans longevity protein DAF-16 by insulin/IGF-1 and germline signaling. Nat. Genet. 28: 139145.

Lithgow, G.J. and Walker, G.A. 2002. Stress resistance as a determinate of C. elegans lifespan. Mech. Ageing Dev. 123: 765-771.

Lotti, L.V., Lanfrancone, L., Migliaccio, E., Zompetta, C., Pelicci, G., Salcini, A.E., Falini, B., Pelicci, P.G., and Torrisi, M.R. 1996. Sch proteins are localized on endoplasmic reticulum membranes and are redistributed after tyrosine kinase receptor activation. Mol. Cell. Biol. 16: 1946-1954.

Luzi, L., Confalonieri, S., Di Fiore, P.P., and Pelicci, P.G. 2000. Evolution of She functions from nematode to human. Curr. Opin. Genet. Dev. 10: 668-674.

Mello, C.C., Kramer, J.M., Stinchcomb, D., and Ambros, V. 1991. Efficient gene transfer in C. elegans: Extrachromosomal maintenance and integration of transforming sequences. EMBO J. 10: 3959-3970.

Migliaccio, E., Giorgio, M., Mele, S., Pelicci, G., Reboldi, P., Pandolfi, P.P., Lanfrancone, L., and Pelicci, P.G. 1999. The p66shc adaptor protein controls oxidative stress response and life span in mammals. Nature 402: 309-313.

Mizuno, T., Hisamoto, N., Terada, T., Kondo, T., Adachi, M., Nishida, E., Kim, D.H., Ausubel, F.M., and Matsumoto, K. 2004. The Caenorhabditis elegans MAPK phosphatase VHP-1 mediates a novel JNK-like signaling pathway in stress response. EMBO J. 23: 2226-2234.

Morris, J.Z., Tissenbaum, H.A., and Ruvkun, G. 1996. A phosphatidylinositol-3-OH kinase family member regulating longevity and diapause in Caenorhabditis elegans. Nature 382: 536-539.

Ogg, S., Paradis, S., Gottlieb, S., Patterson, G.I., Lee, L., Tissenbaum, H.A., and Ruvkun, G. 1997. The Fork head transcription factor DAF-16 transduces insulin-like metabolic and longevity signals in C. elegans. Nature 389: 994-999.

Oh, S.W., Mukhopadhyay, A., Svrzikapa, N., Jiang, F., Davis, R.J., and Tissenbaum, H.A. 2005. JNK regulates lifespan in Caenorhabditis elegans by modulating nuclear translocation of forkhead transcription factor/DAF-16. Proc. Natl. Acad. Sci. 102: 4494-4499.

Okabayashi, Y., Sugimoto, Y., Totty, N.F., Hsuan, J., Kido, Y., Sakaguchi, K., Gout, I., Waterfield, M.D., and Kasuga, M. 1996. Interaction of Shc with adaptor protein adaptins. J. Biol. Chem. 271: 5265-5269.

Paradis, S. and Ruvkun, G. 1998. Caenorhabditis elegans Akt/ PKB transduces insulin receptor-like signals from AGE-1 PI3 kinase to the DAF-16 transcription factor. Genes \& Dev. 12: 2488-2498.

Paradis, S., Ailion, M., Toker, A., Thomas, J.H., and Ruvkun, G. 1999. A PDK1 homolog is necessary and sufficient to transduce AGE-1 PI3 kinase signals that regulate diapause in Caenorhabditis elegans. Genes \& Dev. 13: 1438-1452.

Pelicci, G., Lanfrancone, L., Grignani, F., McGlade, J., Cavallo, F., Forni, G., Nicoletti, I., Grignani, F., Pawson, T., and Pelicci, P.G. 1992. A novel transforming protein (SHC) with an $\mathrm{SH} 2$ domain is implicated in mitogenic signal transduction. Cell 70: 93-104.

Pinton, P., Rimessi, A., Marchi, S., Orsini, F., Migliaccio, E., Giorgio, M., Contursi, C., Minucci, S., Mantovani, F., Wieckowski, M.R., et al. 2007. Protein kinase C $\beta$ and prolyl isomerase 1 regulate mitochondrial effects of the life-span determinant p66Shc. Science 315: 659-663.

Piper, M.D., Selman, C., McElwee, J.J., and Partridge, L. 2008. Separating cause from effect: How does insulin/IGF signalling control lifespan in worms, flies and mice? J. Intern. Med. 263: 179-191.

Skolnik, E.Y., Batzer, A., Li, N., Lee, C.H., Lowenstein, E., Mohammadi, M., Margolis, B., and Schlessinger, J. 1993. The function of GRB2 in linking the insulin receptor to Ras signaling pathways. Science 260: 1953-1955.

Tatar, M., Kopelman, A., Epstein, D., Tu, M.P., Yin, C.M., and Garofalo, R.S. 2001. A mutant Drosophila insulin receptor homolog that extends life-span and impairs neuroendocrine function. Science 292: 107-110.

Tsiokas, L., Kim, E., Arnould, T., Sukhatme, V.P., and Walz, G. 1997. Homo- and heterodimeric interactions between the gene products of PKD1 and PKD2. Proc. Natl. Acad. Sci. 94: 6965-6970.

Villanueva, A., Lozano, J., Morales, A., Lin, X., Deng, X., Hengartner, M.O., and Kolesnick, R.N. 2001. jkk-1 and mek-1 regulate body movement coordination and response to heavy metals through jnk-1 in Caenorhabditis elegans. EMBO $J$. 20: 5114-5128.

Wang, M.C., Bohmann, D., and Jasper, H. 2003. JNK signaling confers tolerance to oxidative stress and extends lifespan in Drosophila. Dev. Cell 5: 811-816.

Yanase, S., Yasuda, K., and Ishii, N. 2002. Adaptive responses to oxidative damage in three mutants of Caenorhabditis elegans (age-1, mev-1 and daf-16) that affect life span. Mech. Ageing Dev. 123: 1579-1587.

Yukimasa, S., Masaki, T., Yoshida, S., Uchida, N., Watanabe, S., Usuki, H., Yoshiji, H., Maeta, T., Ebara, K., Nakatsu, T., et al. 2005. Enhanced expression of p46 Shc in the nucleus and p52 Shc in the cytoplasm of human gastric cancer. Int. J. Oncol. 26: 905-911.

Zhou, M.M., Ravichandran, K.S., Olejniczak, E.F., Petros, A.M., Meadows, R.P., Sattler, M., Harlan, J.E., Wade, W.S., Burakoff, S.J., and Fesik, S.W. 1995. Structure and ligand recognition of the phosphotyrosine binding domain of Shc. Nature 378: $584-592$. 


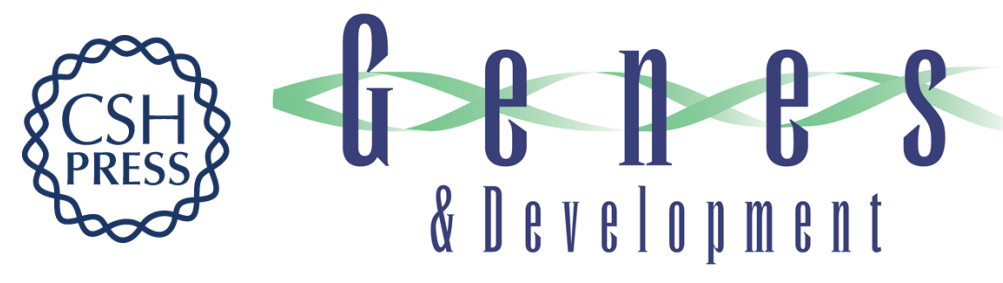

\section{SHC-1/p52Shc targets the insulin/IGF-1 and JNK signaling pathways to modulate life span and stress response in $C$. elegans}

Elke Neumann-Haefelin, Wenjing Qi, Elisabeth Finkbeiner, et al.

Genes Dev. 2008, 22:

Access the most recent version at doi:10.1101/gad.478408

Supplemental
Material http://genesdev.cshlp.org/content/suppl/2008/09/22/22.19.2721.DC1

References This article cites 55 articles, 30 of which can be accessed free at:

http://genesdev.cshlp.org/content/22/19/2721.full.html\#ref-list-1

License

Email Alerting Receive free email alerts when new articles cite this article - sign up in the box at the top

Service

right corner of the article or click here.

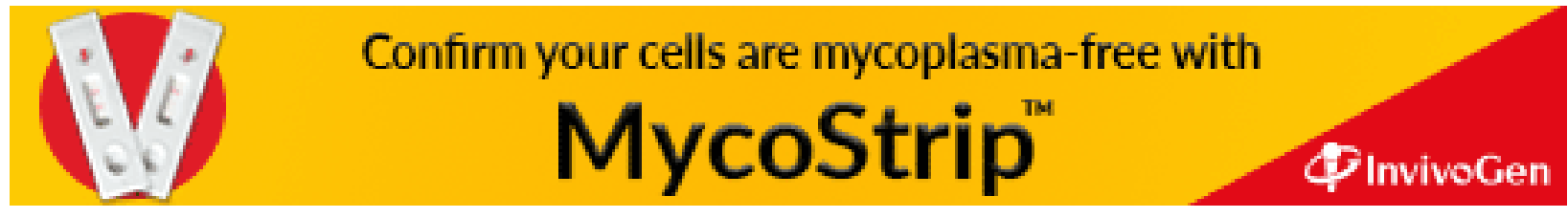

